\title{
Analysis of shared heritability in common disorders of the brain
}

\section{Brainstorm Consortium}

2018-06-22

Brainstorm Consortium , Anttila, V, Bulik-Sullivan, B, Finucane, H K, Walters, R K, Bras , J , Duncan , L , Escott-Price , V , Falcone , G J , Gormley , P, Malik , R, Patsopoulos , N A , Ripke , S , Wei , Z , Yu , D , Lee , P H, Turley , P, Grenier-Boley , B , Chouraki , V , Kamatani , Y, Berr , C , Letenneur , L, Hannequin , D , Amouyel , P , Boland , A, Deleuze , J-F , Duron , E , Vardarajan , B N , Reitz , C , Goate , A M , Huentelman , M J , Kamboh , M I , Larson, E B , Rogaeva, E , St George-Hyslop , P , Hakonarson , H , Kukull , W A, Palta , P, Wedenoja , J , Artto , V , Kaunisto , M , Vepsäläinen , S, Kurki , M I , Hämäläinen , E , Kaprio , J , Metspalu , A , Keski-Rahkonen , A , Raevuori , A , Ripatti , S , Lönnqvist , J , Daly , M , Palotie , A \& Neale , B M 2018 , ' Analysis of shared heritability in common disorders of the brain ' , Science , vol. 360 , no. 6395 , 8757 , pp. 1313-+ . https://doi.org/10.1126/science.aap8757

http://hdl.handle.net/10138/303879

https://doi.org/10.1126/science.aap8757

publishedVersion

Downloaded from Helda, University of Helsinki institutional repository.

This is an electronic reprint of the original article.

This reprint may differ from the original in pagination and typographic detail.

Please cite the original version. 
RESEARCH ARTICLE SUMMARY

PSYCHIATRIC GENOMICS

\section{Analysis of shared heritability in common disorders of the brain}

\author{
The Brainstorm Consortium $\dagger$
}

INTRODUCTION: Brain disorders may exhibit shared symptoms and substantial epidemiological comorbidity, inciting debate about their etiologic overlap. However, detailed study of phenotypes with different ages of onset, severity, and presentation poses a considerable challenge. Recently developed heritability methods allow us to accurately measure correlation of genome-wide common variant risk between two phenotypes from pools of different individuals and assess how connected they, or at least their genetic risks, are on the genomic level. We used genome-wide association data for 265,218 patients and 784,643 control participants, as well as 17 phenotypes from a total of 1,191,588 individuals, to quantify the degree of overlap for genetic risk factors of 25 common brain disorders.

RATIONALE: Over the past century, the classification of brain disorders has evolved to reflect the medical and scientific communities' assessments of the presumed root causes of clinical phenomena such as behavioral change, loss of motor function, or alterations of consciousness. Directly observable phenomena (such as the presence of emboli, protein tangles, or unusual electrical activity patterns) generally define and separate neurological disorders from psychiatric disorders. Understanding the genetic underpinnings and categorical distinctions for brain disorders and related phenotypes may inform the search for their biological mechanisms.

RESULTS: Common variant risk for psychiatric disorders was shown to correlate significantly, especially among attention deficit hyperactivity disorder (ADHD), bipolar disorder, major depressive disorder (MDD), and schizophrenia. By contrast, neurological disorders appear more distinct from one another and from the psychiatric disorders, except for migraine, which was significantly correlated to ADHD, MDD, and Tourette syndrome. We demonstrate that, in the general population, the personality trait neuroticism is significantly correlated with almost every psychiatric disorder and migraine. We also identify significant genetic sharing between disorders and early life cognitive measures (e.g., years of education and college attainment) in the general population, demonstrating positive correlation with several psychiatric disorders (e.g., anorexia nervosa and bipolar disorder) and negative correlation with several neurological phenotypes (e.g., Alzheimer's disease and ischemic stroke), even though the latter are considered to result from specific processes that occur later in life. Extensive simulations were also performed to inform how statistical power, diagnostic misclassification, and phenotypic heterogeneity influence genetic correlations.

CONCLUSION: The high degree of genetic correlation among many of the psychiatric disorders adds further evidence that their current clinical boundaries do not reflect distinct underlying pathogenic processes, at least on the genetic level. This suggests a deeply intercon-

\section{ON OUR WEBSITE}

Read the full article at http://dx.doi.

org/10.1126/

science.aap8757

\section{......................... analyses may provide im-}

portant "scaffolding" to support such restructuring of psychiatric nosology, which likely requires incorporating many levels of information. By contrast, we find limited evidence for widespread common genetic risk sharing among neurological disorders or across neuthat both psychiatric and neurological disorders have robust correlations with cognitive and personality measures. Further study is needed to evaluate whether overlapping genetic contributions to psychiatric pathology may influence treatment choices. Ultimately, such developments may pave the way toward reduced heterogeneity and improved diagnosis and treatment of psychiatric disorders.

The list of author affiliations is available in the full article online. †Corresponding authors: Verneri Anttila (verneri.anttila@gmail. com); Aiden Corvin (acorvin@tcd.ie); Benjamin M. Neale (bneale@broadinstitute.org)

Cite this article as The Brainstorm Consortium, Science 360, eaap8757 (2018). DOI: 10.1126/science.aap8757 rological and psychiatric disorders. We show
Psychiatric/quantitative

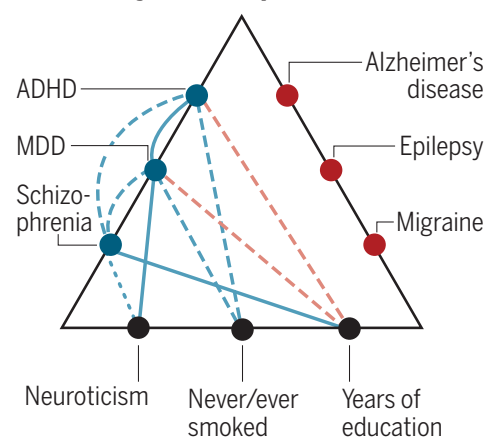

Psychiatric/neurological

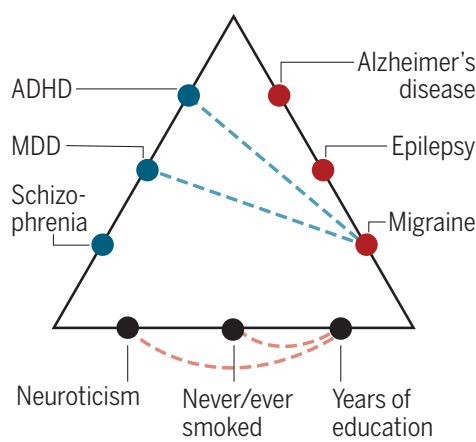

Neurological/quantitative

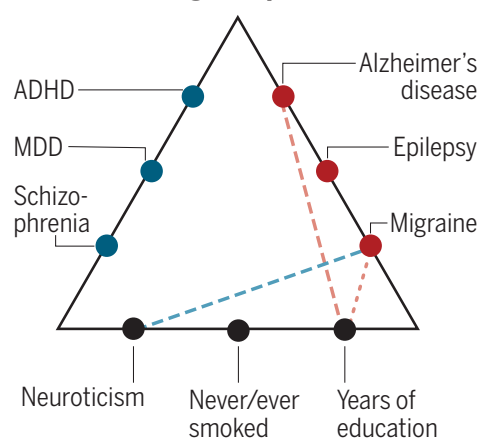

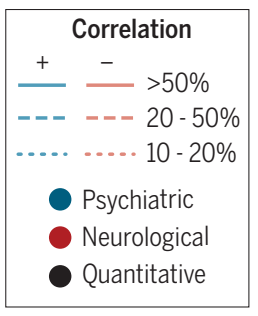

Subsection of genetic risk correlations among brain disorders and quantitative phenotypes. Heritability analysis of brain disorders points to pervasive sharing of genetic risk among psychiatric disorders. These correlations are largely absent among neurological disorders but are present for both groups in relation to neurocognitive quantitative phenotypes. Only significant correlations shown. Line color and solidity indicate direction and magnitude of correlation, respectively. 
RESEARCH ARTICLE

PSYCHIATRIC GENOMICS

\section{Analysis of shared heritability in common disorders of the brain}

\author{
The Brainstorm Consortium* ${ }^{*}$
}

Disorders of the brain can exhibit considerable epidemiological comorbidity and often share symptoms, provoking debate about their etiologic overlap. We quantified the genetic sharing of 25 brain disorders from genome-wide association studies of 265,218 patients and 784,643 control participants and assessed their relationship to 17 phenotypes from 1,191,588 individuals. Psychiatric disorders share common variant risk, whereas neurological disorders appear more distinct from one another and from the psychiatric disorders. We also identified significant sharing between disorders and a number of brain phenotypes, including cognitive measures. Further, we conducted simulations to explore how statistical power, diagnostic misclassification, and phenotypic heterogeneity affect genetic correlations. These results highlight the importance of common genetic variation as a risk factor for brain disorders and the value of heritability-based methods in understanding their etiology.

I he classification of brain disorders has evolved over the past century, reflecting the medical and scientific communities' assessments of the presumed root causes of clinical phenomena such as behavioral change, loss of motor function, spontaneous movements, or alterations of consciousness. Directly observable phenomena (such as the presence of emboli, protein tangles, or unusual electrical activity patterns) generally define and separate neurological disorders from psychiatric disorders (1). Understanding the genetic underpinnings and categorical distinctions between brain disorders may be helpful in informing the search for the biological pathways underlying their pathophysiology $(2,3)$.

Studies of twins and families have indicated that, in general, brain disorders (excepting those caused by trauma, infection, or cancer) show substantial heritability (4). Epidemiological and twin studies have explored patterns of phenotypic overlaps (5-7), and comorbidity has been reported for many pairs of disorders, including bipolar disorder and migraine (8), stroke and major depressive disorder (MDD) (9), epilepsy and autism spectrum disorder (ASD), and epilepsy and attention deficit hyperactivity disorder (ADHD) $(10,11)$. Furthermore, direct etiological links may also exist-e.g., mutations in the same ion channel genes confer pleiotropic risk for multiple distinct brain phenotypes (12-14). Genome-wide association studies (GWASs) have demonstrated that individual common risk variants can overlap across traditional diagnostic boundaries $(15,16)$ and that disorders such as schizophrenia, MDD, and bipolar disorder can have genetic correlations (17).

*All authors with their affiliations are listed at the end of this paper. tCollaborators and affiliations are listed in the supplementary materials.
GWASs have also demonstrated that common genetic variation contributes to the heritability of brain disorders. Generally, this occurs via the combination of many common variants-examples include Alzheimer's disease (18), bipolar disorder (19), migraine (20), Parkinson's disease (21), and schizophrenia (22)-each with a small individual effect. In addition to locus discovery, the degree of distinctiveness (23) across neurological and psychiatric phenotypes can be evaluated with the introduction of novel heritability-based methods (24) and sufficiently large sample sizes for robust heritability analysis. These analyses can shed light on the nature of these diagnostic boundaries and explore the extent of shared common variant genetic influences.

\section{Study design}

The Brainstorm Consortium, a collaboration among GWAS meta-analysis consortia for 25 disorders hensive heritability and correlation analysis of brain disorders. We included meta-analyses of any common brain disorders for which we could identify a GWAS meta-analysis consortium of sufficient size for heritability analysis. The total study sample consists of 265,218 cases of different brain disorders and 784,643 controls (Table 1 ) and includes at least one representative of most ICD-10 (10th revision of the International Statistical Classification of Diseases and Related Health Problems) blocks covering mental and behavioral disorders and diseases of the central nervous system (CNS). Also included are 1,191,588 samples for 13 behavioral-cognitive phenotypes ( $n=744,486$ individuals) traditionally viewed as brain-related, as well as 4 additional phenotypes ( $n=447,102$ individuals) selected to represent known, well-delineated etiological processes immune disorders (Crohn's disease), vascular disease (Table 1), was assembled to perform a compre- (coronary artery disease), and anthropomorphic measures [height and body mass index (BMI)]\} (Table 2).

GWAS summary statistics for the 42 disorders and phenotypes were centralized and underwent uniform quality control and processing (25). To avoid potential bias arising from ancestry differences, we used European-only metaanalyses for each disorder and generated new meta-analyses for those datasets where the original sample sets had diverse ancestries. Clinically relevant subtypes from three disorders (epilepsy, migraine, and ischemic stroke) were also included; in these cases, the subtype datasets are parts of the top-level dataset (Table 1).

We have developed a heritability estimation method, linkage disequilibrium score (LDSC) regression (24), which was used to calculate heritability estimates and correlations, as well as to estimate their statistical significance from block jackknife-based standard errors. More formally, we estimate the common variant heritability $\left(h^{2} g\right)$ of each disorder, defined as the proportion of phenotypic variance in the population that could theoretically be explained by an optimal linear predictor formed using the additive effects of all common (minor allele frequency $>5 \%$ ) autosomal single-nucleotide polymorphisms (SNPs). The genetic correlation for a pair of phenotypes is then defined as the correlation between their optimal genetic predictors. Heritability for binary disorders and phenotypes was transformed to the liability scale. We further performed a weighted least-squares regression analysis to evaluate whether differences relating to study makeup (such as sample size) were correlated with the magnitude of the correlation estimates. Finally, we performed a heritability partitioning analysis (25) by means of stratified LD score regression to examine whether the observed heritability for the disorders or phenotypes was enriched into any of the tissue-specific regulatory regions or functional category partitions of the genome, using 10 top-level tissue-type and 53 functional partitions from Finucane et al. (26). Simulated phenotype data was then generated under different scenarios by permuting 120,267 genotyped individuals from the UK Biobank (25) to evaluate statistical power and aid in interpreting the results (25).

\section{Heritability estimates and their error sources}

We observed a similar range of heritability estimates among the disorders and the behavioralcognitive phenotypes (fig. S1, A and B, and table S1 and S2), roughly in line with previously reported estimates from smaller datasets (table S3). Three ischemic stroke subtypes (cardioembolic, large-vessel disease, and small-vessel disease) as well as the "agreeableness" personality measure from the NEO Five-Factor Inventory (27) had insufficient evidence of additive heritability for robust analysis and thus were excluded from further examination (25). The only observed correlation between heritability estimates and factors relating to study makeup (table S4 and fig. S1, 
Table 1. Brain disorder phenotypes used in the Brainstorm project. Indented phenotypes are part of a larger whole (e.g., the epilepsy study contains the samples from both focal epilepsy and generalized epilepsy). "Anxiety disorders" refers to a meta-analysis of five subtypes (generalized anxiety disorder, panic disorder, social phobia, agoraphobia, and specific phobias). References are listed in table S1 and data availability in table S13. PGC-ADD2, Psychiatric Genomics Consortium (PGC) Attention Deficit Disorder Working Group; PGC-ED, PGC Eating Disorder Working Group; ANGST, Anxiety Neuro Genetics STudy; PGC-AUT, PGC Autism Spectrum Disorder Working Group; PGC-BIP2, PGC Bipolar Disorder Working Group;
PGC-MDD2, PGC Major Depressive Disorder Working Group; PGC-OCDTS, PGC Obsessive Compulsive Disorder and Tourette Syndrome Working Group; PGC-PTSD, PGC Posttraumatic Stress Disorder Working Group; PGC-SCZ2, PGC Schizophrenia Working Group; IGAP, International Genomics of Alzheimer's Project; ILAE, International League Against Epilepsy Consortium on Complex Epilepsies; ISGC, International Stroke Genetics Consortium; METASTROKE, a consortium of the ISGC; IHGC, International; Headache Genetics Consortium; IMSGC, International Multiple Sclerosis Genetics Consortium; IPDGC, International Parkinson's Disease Genomics Consortium. " indicates same as above.

\begin{tabular}{|c|c|c|c|c|c|c|c|}
\hline \multicolumn{4}{|c|}{ Psychiatric disorders } & \multicolumn{4}{|c|}{ Neurological disorders } \\
\hline Disorder & Source & Cases & Controls & Disorder & Source & Cases & Controls \\
\hline $\begin{array}{l}\text { Attention deficit } \\
\text { hyperactivity disorder }\end{array}$ & PGC-ADD2 & 12,645 & 84,435 & Alzheimer's disease & IGAP & 17,008 & 37,154 \\
\hline Anorexia nervosa & PGC-ED & 3495 & 10,982 & Epilepsy & ILAE & 7779 & 20,439 \\
\hline Anxiety disorders & ANGST & 5761 & 11,765 & Focal epilepsy & $"$ & $4601^{*}$ & $17,985^{*}$ \\
\hline Autism spectrum disorder & PGC-AUT & 6197 & 7377 & Generalized epilepsy & $"$ & $2525^{*}$ & $16,244^{*}$ \\
\hline Bipolar disorder & PGC-BIP2 & 20,352 & 31,358 & Intracerebral hemorrhage & ISGC & 1545 & 1481 \\
\hline Major depressive disorder & PGC-MDD2 & 66,358 & 153,234 & Ischemic stroke & METASTROKE & 10,307 & 19,326 \\
\hline $\begin{array}{l}\text { Obsessive-compulsive } \\
\text { disorder }\end{array}$ & PGC-OCDTS & 2936 & 7279 & Cardioembolic stroke & $"$ & $1859^{*}$ & $17,708^{*}$ \\
\hline Posttraumatic stress disorder & PGC-PTSD & 2424 & 7113 & Early onset stroke & $"$ & $3274^{*}$ & $11,012^{*}$ \\
\hline Schizophrenia & PGC-SCZ2 & 33,640 & 43,456 & Large-vessel disease & "... & $1817^{*}$ & $17,708^{*}$ \\
\hline \multirow[t]{6}{*}{ Tourette syndrome } & PGC-OCDTS & 4220 & 8994 & Small-vessel disease & $"$ & $1349^{*}$ & $17,708^{*}$ \\
\hline & & & & Migraine & IHGC & 59,673 & 316,078 \\
\hline & & & & Migraine with aura & " & $6332^{*}$ & $142,817^{*}$ \\
\hline & & & & Migraine without aura & $"$ & $8348^{*}$ & $136,758^{*}$ \\
\hline & & & & Multiple sclerosis & IMSGC & 5545 & 12,153 \\
\hline & & & & Parkinson's disease & IPDGC & 5333 & 12,019 \\
\hline Total psychiatric & & 158,028 & 365,993 & Total neurologic & & 107,190 & 418,650 \\
\hline
\end{tabular}

*Sample count for a phenotype that is part of a larger group.

$\mathrm{C}$ to $\mathrm{F}$ ) was a modest correlation between age of disorder onset and heritability, suggesting that early onset brain disorders tend to be more heritable. Because some of our interpretation of the results depends on lack of observed correlation, we explored the behavior of observed correlation versus power (fig. S2A), standard errors (fig. S2B), and the individual results (fig. S2, C and D) to identify where we can be reasonably robust in claiming lack of correlation.

The common variant heritability estimates for the psychiatric and neurological disorders were generally somewhat lower than previously reported estimates from common variants (table S5). When comparing estimates reported here with those previously reported in studies with smaller sample sizes (28), a similar pattern was observed for the behavioral-cognitive traits, with the exception of "openness," "neuroticism," and "never/ever smoked" (defined as those who have never smoked versus those who have smoked at some point) suggesting that some attenuation in heritability is observed when moving to larger sample sizes. Measures related to cognitive ability, such as childhood cognitive performance [heritability estimate of 0.19 (SE: 0.03)] and years of education [heritability estimate of 0.30 (SE: $0.01)$ ], yielded estimates that were more consistent with previous estimates of the herita- bility of intelligence $(29,30)$, suggesting that the cognitive measures may be less prone to phenotypic measurement error and/or have a higher heritability overall than the personality measures.

These heritability estimates should be interpreted somewhat cautiously, as they reflect the phenotype ascertained in each study and will be deflated in the presence of diagnostic heterogeneity, ascertainment errors, or unusual contributions of high-impact rare variants. To evaluate potential sources of these differences, we explored three approaches (25): evaluating the differences in real data, simulation work (table S5), and quantifying the magnitude of effect for potentially implied misclassification (table S6).

In comparison with heritability estimates obtained using twin and family data, the more diverse selection and survival biases in the underlying data may attenuate the heritability estimates and correlations, as may increased within-disorder heterogeneity introduced by the larger metaanalyses. A related explanation for the lower estimates of heritability may be that increasing sample sizes have led to expanded inclusion criteria, meaning that less severely affected cases with a lower overall burden of risk factors (both genetic and environmental) might be included, which in turn would attenuate estimates of heritability. However, the successful identification of genome-wide significant loci suggests that these larger samples are nevertheless very useful for genetic studies, and the simulation results suggest that this has, at most, a limited effect on estimated genetic correlations (fig. S9). Even so, some of the pairs of phenotypes included here lack sufficient power for robust estimation of genetic correlations. Moreover, our analyses examine only the properties of common variant contributions; extending these analyses to include the effects of rare variants may further inform the extent of genetic overlap. For example, epilepsy and ASD show substantial overlap in genetic risk from de novo loss-of-function mutations (31), in contrast to the limited common variant sharing observed in this study. This may suggest that the rare and common variant contributions to genetic overlap may behave differently and that incorporating the two variant classes into a single analysis may provide further insight into brain disorder pathogenesis.

To address the possibility of methodological differences contributing to the differences in the estimates, and although LDSC and GREML have previously been shown to yield similar estimates from the same data (24), we performed our own comparison in Alzheimer's disease data (32) (selected on the basis of data availability). In Alzheimer's disease, the previously published heritability estimate [0.24 (SE: 0.03)] is significantly different 
Table 2. Behavioral-cognitive and additional phenotypes used in the study. Indented phenotypes are part of a larger whole (e.g., samples in the college attainment analysis are a subset of those in the analysis for years of education). (d), dichotomous phenotype; (q), quantitative phenotype. References and phenotype definitions are listed in table S2, and data availability in table S13. SSGAC, Social Science Genetic Association Consortium; CTG, Complex Trait Genetics Lab; GPC, Genetics of Personality Consortium; TAG, Tobacco and Genetics Consortium; GIANT, Genetic Investigation of ANthropometric Traits consortium; Cardiogram, CARDloGRAMplusC4D Consortium; IIBDGC, International Inflammatory Bowel Disease Genetics Consortium.

Phenotype

Source

Samples

\begin{tabular}{|c|c|c|}
\hline \multicolumn{3}{|c|}{ Behavioral-cognitive phenotypes } \\
\hline \multicolumn{3}{|l|}{ Cognitive } \\
\hline Years of education (q) & SSGAC & 293,723 \\
\hline College attainment $(d)$ & $"$ & $120,917^{*}$ \\
\hline Cognitive performance $(q)$ & " & $17,989 *$ \\
\hline Intelligence (d) & CTG & 78,308 \\
\hline \multicolumn{3}{|l|}{ Personality measures } \\
\hline Subjective well-being & SSGAC & 298,420 \\
\hline Depressive symptoms & " & $161,460^{*}$ \\
\hline Neuroticism (q) & "..... & $170,911^{*}$ \\
\hline Extraversion (q) & GPC & $63,030^{*}$ \\
\hline Agreeableness (q) & "......... & $17,375^{*}$ \\
\hline Conscientiousness (q) & $"$ & $17,375^{*}$ \\
\hline Openness (q) & ".... & $17,375^{*}$ \\
\hline \multicolumn{3}{|l|}{ Smoking-related } \\
\hline Never/ever smoked $(d)$ & TAG & 74,035 \\
\hline Cigarettes per day (q) & TAG & $38,617^{*}$ \\
\hline \multicolumn{3}{|c|}{ Additional phenotypes } \\
\hline $\mathrm{BMI}(\mathrm{q})$ & GIANT & 339,224 \\
\hline Height (q) & " & $253,288^{*}$ \\
\hline Coronary artery disease (d) & Cardiogram & 86,995 \\
\hline Crohn's disease & IIBDGC & 20,883 \\
\hline Total & & $1,124,048$ \\
\hline
\end{tabular}

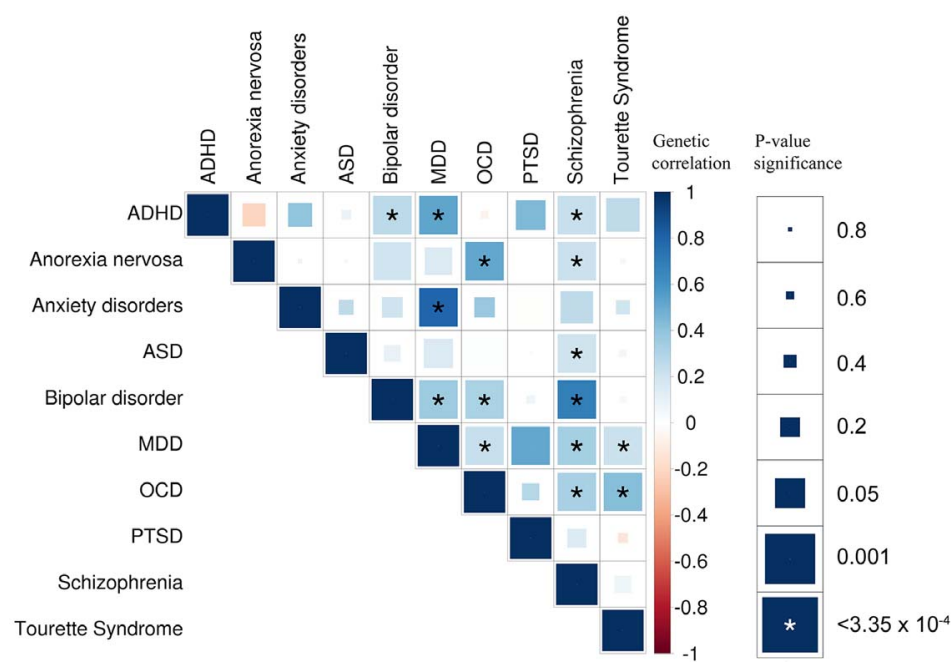

Fig. 1. Genetic correlations across psychiatric phenotypes. The color of each box indicates the magnitude of the correlation, and the size of the box indicates its significance (LDSC), with significant correlations filling each square completely. Asterisks indicate genetic correlations that are significantly different from zero after Bonferroni correction. from the estimate in the current study [0.13 (SE: 0.02)]. These differences may reflect implicit heterogeneity in a much larger case collection used in the current study (effective sample size 10,494 versus 46,669 ) and the potential reasons listed above, but they could also be due to methodological variability (most of the previous approximations listed in table S3 are estimated with a different methodology). To evaluate this, we applied the same analytical process used in this paper to the summary statistics of the GERAD (Genetic and Environmental Risk in Alzheimer's Disease) cohort (3941 cases and 7848 controls) from the Alzheimer's disease meta-analysis, where the previous heritability estimate was calculated. There, we obtained a heritability estimate of 0.25 (SE: 0.04), which agrees closely with the published estimate of 0.24 (SE: 0.03), suggesting that the different approximations may reflect differences between datasets rather than methodological variability.

\section{Correlations among brain disorders}

We observed widespread sharing across psychiatric disorders (Fig. 1 and fig. S3) by expanding the number of brain disorder pairs studied beyond those previously reported (17), but similar sharing was not observed among neurological disorders. Among the psychiatric disorders, schizophrenia showed significant genetic correlation with most of the psychiatric disorders, whereas MDD was positively (though not necessarily significantly) correlated with every other disorder tested. Further, schizophrenia, bipolar disorder, anxiety disorders, MDD, and $\mathrm{ADHD}$ each showed a high degree of correlation to the four others [average genetic correlation $\left(r_{\mathrm{g}}\right)=0.40$ ] (table S7A). Anorexia nervosa, obsessive-compulsive disorder (OCD), and schizophrenia also demonstrated significant sharing among themselves (Fig. 1), as did Tourette syndrome (TS), OCD, and MDD, as well as ASD and schizophrenia. Posttraumatic stress disorder (PTSD) showed no significant correlation with any of the other psychiatric phenotypes (though some correlation to ADHD and MDD was observed), and both ASD and TS appear to potentially be more distinct from the other psychiatric disorders. The modest power of the ASD, PTSD, and TS meta-analyses, however, limits the strength of this conclusion (fig. S2C).

Neurological disorders showed a more limited extent of genetic correlation than that of the psychiatric disorders (Fig. 2, fig. S4, and table S7A), suggesting greater diagnostic specificity and/or more distinct etiologies. Parkinson's disease, Alzheimer's disease, generalized epilepsy, and multiple sclerosis (MS) showed little to no correlation with other brain disorders. The highest degree of genetic correlation among the neurological disorders was observed for focal epilepsy (average $r_{\mathrm{g}}=0.46$, excluding the other epilepsy datasets), though none of the correlations were significant, reflecting the relatively modest power of the current focal epilepsy meta-analysis (fig. S2C). However, the modest heritability and the broad pattern of sharing observed for focal epilepsy may be consistent with heterogeneity and 
potentially even diagnostic misclassification across a range of neurological conditions.

In the cross-category correlation analysis, the observed pattern is consistent with limited sharing across the included neurological and psychiatric disorders (Fig. 3; average $r_{\mathrm{g}}=0.03$ ). The only significant cross-category correlations were with migraine, suggesting that this disorder may share some of its genetic architecture with psychiatric disorders: migraine and $\mathrm{ADHD}\left(r_{\mathrm{g}}=0.26, P=\right.$ $\left.8.81 \times 10^{-8}\right)$, migraine and TS $\left(r_{\mathrm{g}}=0.19, P=1.80 \times\right.$ $\left.10^{-5}\right)$, and migraine and $\mathrm{MDD}\left(r_{\mathrm{g}}=0.32, P=\right.$ $1.42 \times 10^{-22}$ for all migraine; $r_{\mathrm{g}}=0.23, P=5.23 \times$ $10^{-5}$ for migraine without aura; $r_{\mathrm{g}}=0.28, P=1.00 \times$ $10^{-4}$ for migraine with aura).

We observed several significant genetic correlations between the behavioral-cognitive or additional phenotypes and brain disorders (Fig. 4 and table S7B). Results for cognitive traits were dichotomous among psychiatric phenotypes (fig. S5A), with ADHD, anxiety disorders, MDD, and TS showing negative correlations to the cognitive measures and anorexia nervosa, ASD, bipolar disorder, and OCD showing positive correlations. Schizophrenia showed more mixed results, with a significantly negative correlation to intelligence but a positive correlation to years of education. Among neurological phenotypes (fig. S5B), the correlations were either negative or null, with Alzheimer's disease, epilepsy, intracerebral hemorrhage (ICH), ischemic stroke, early onset stroke, and migraine showing significantly negative correlations. Correlations between college attainment and years of education with bipolar disorder (24), Alzheimer's disease, and schizophrenia have been previously reported (33).

Among the personality and symptom measures, significant positive correlations were observed between neuroticism and anorexia nervosa, anxiety disorders, migraine, migraine without aura, MDD, OCD, schizophrenia, and TS [fig. S6, A and $\mathrm{B}$; replicating previously reported correlations with MDD and schizophrenia (34)]; between depressive symptoms and ADHD, anxiety disorder, bipolar disorder, MDD, and schizophrenia; and between subjective well-being and anxiety disorder, bipolar disorder, and MDD. For smokingrelated measures, the only significant genetic correlations were between never/ever smoked and $\operatorname{MDD}\left(r_{\mathrm{g}}=0.33, P=3.10 \times 10^{-11}\right)$ as well as $\operatorname{ADHD}\left(r_{\mathrm{g}}=0.37, P=3.15 \times 10^{-6}\right)$.

Among the additional phenotypes, the two examples of disorders with well-defined etiologies had different results. Crohn's disease, representing immunological pathophysiology, showed no correlation with any of the study phenotypes, whereas the phenotype representing vascular pathophysiology (coronary artery disease) showed significant correlation to MDD $\left(r_{\mathrm{g}}=0.19, P=\right.$ $8.71 \times 10^{-5}$ ) as well as the two stroke-related phenotypes $\left(r_{\mathrm{g}}=0.69, P=2.47 \times 10^{-6}\right.$ to ischemic stroke and $r_{\mathrm{g}}=0.86, P=2.26 \times 10^{-5}$ to early onset stroke), suggesting shared genetic effects across these phenotypes. Significant correlations were also observed for BMI, which was positively correlated with $\mathrm{ADHD}$ and $\mathrm{MDD}$, and negatively correlated with anorexia nervosa [as previous-

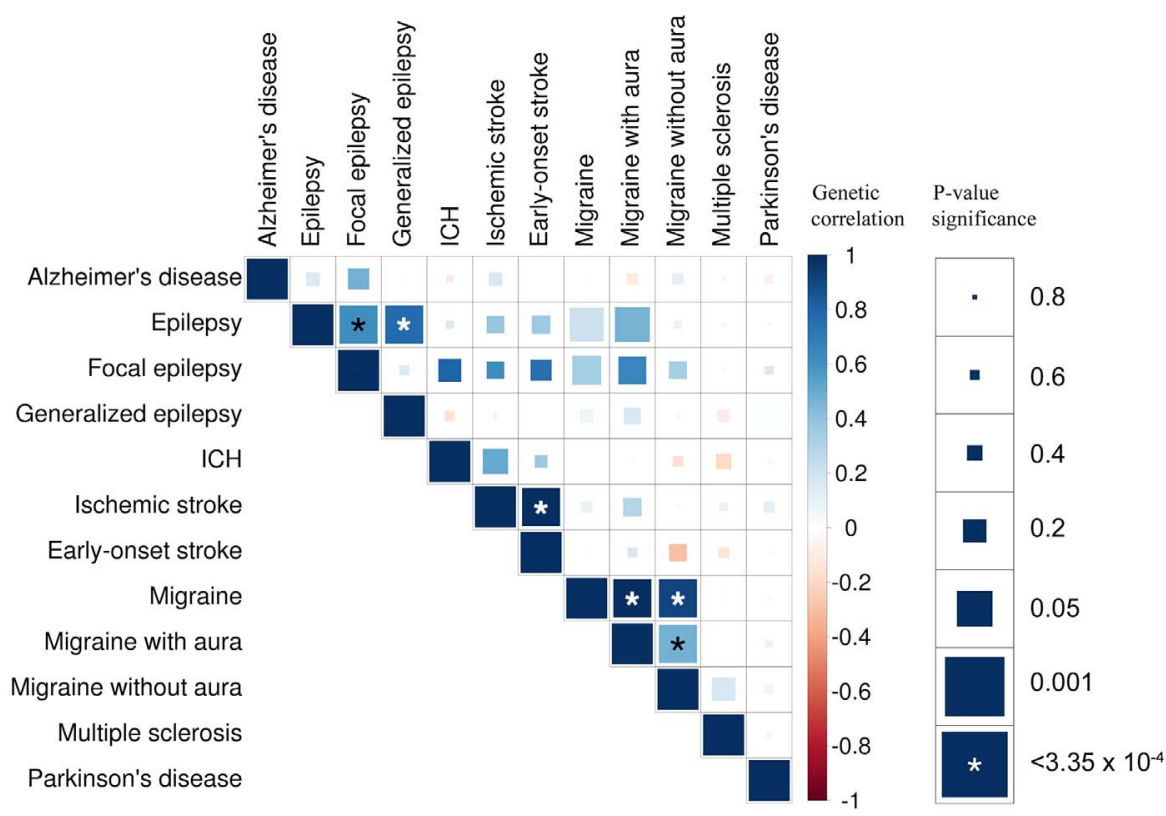

Fig. 2. Genetic correlations across neurological phenotypes. The color of each box indicates the magnitude of the correlation, and the size of the box indicates its significance (LDSC), with significant correlations filling each square completely. Asterisks indicate genetic correlations that are significantly different from zero after Bonferroni correction. Some phenotypes have substantial overlaps (Table 1)-for instance, all cases of generalized epilepsy are also cases of epilepsy. Asterisks indicate significant genetic correlation after multiple testing correction.

ly reported with a different dataset (24)] and schizophrenia.

Our enrichment analysis (fig. S7 and tables S8 to S12) demonstrated significant heritability enrichments between the CNS and generalized epilepsy, MDD, TS, college attainment, intelligence, neuroticism, and the never/ever smoked trait; between depressive symptoms and adrenal/ pancreatic cells and tissues; as well as between hematopoietic cells (including immune system cells) and MS (fig. S7, A and B, and tables S8 and S9). We replicated the reported (CNS) enrichment for schizophrenia, bipolar disorder, and years of education (tables S8 and S9) and observed the reported enrichments for BMI (CNS), years of education (CNS), height (connective tissues and bone, cardiovascular system, and other), and Crohn's disease (hematopoietic cells) from the same datasets (fig. S7, C and D) (26). The psychiatric disorders with large numbers of identified GWAS loci (bipolar disorder, MDD, and schizophrenia) and migraine, which was the only crosscorrelated neurological disorder, show enrichment to conserved regions (tables S10 and S12), whereas the other neurological disorders with similar numbers of loci (MS, Alzheimer's disease, and Parkinson's disease) do not (fig. S7, A and B). Enrichment to conserved regions was also observed for neuroticism, intelligence, and college attainment and to H3K9ac peaks for BMI (tables S11 and S12).

\section{Discussion}

By integrating and analyzing the genome-wide association summary statistic data from consor- tia of 25 brain disorders, we find that psychiatric disorders broadly share a considerable portion of their common variant genetic risk, especially across schizophrenia, MDD, bipolar disorder, anxiety disorder, and ADHD, whereas neurological disorders are more genetically distinct. Across categories, psychiatric and neurologic disorders share relatively little common genetic risk, suggesting that multiple different and largely independently regulated etiological pathways may give rise to similar clinical manifestations [e.g., psychosis, which manifests in both schizophrenia (35) and Alzheimer's disease (36)]. Except for migraine, which appears to share some genetic architecture with psychiatric disorders, the existing clinical delineation between neurology and psychiatry is corroborated at the level of common variant risk for the studied disorders.

On the basis of the observed results, we performed some exploratory analyses to address concerns about diagnostic overlap and misclassification, which are particularly relevant to psychiatric disorders, owing to their spectral nature. Given that the broad and continuous nature of psychiatric disorder spectra has long been clinically recognized (37-39) and that patients can, in small numbers, progress from one diagnosis to another (40), we evaluated to what extent this kind of diagnostic overlap could explain the observed correlations. Genetic correlation could arise if, for example, patients progress through multiple diagnoses over their lifetime or if some specific diagnostic boundaries between phenotype pairs are particularly porous to misclassification (table S5). Although, for instance, migraine and 


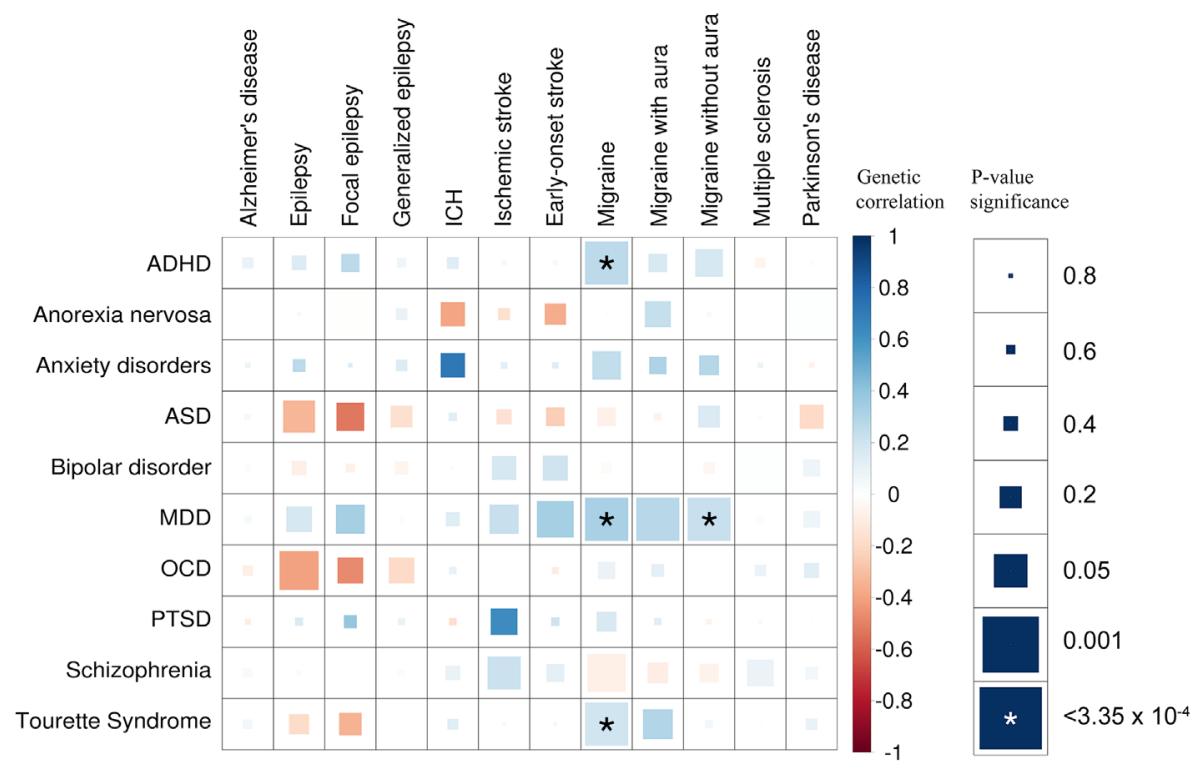

Fig. 3. Genetic correlations across neurological and psychiatric phenotypes. The color of each box indicates the magnitude of the correlation, and the size of the box indicates its significance (LDSC), with significant correlations filling each square completely. Asterisks indicate genetic correlations that are significantly different from zero after Bonferroni correction.

schizophrenia are unlikely to be mistaken for one another, there may be more substantial misclassification between particular psychiatric disorders, consistent with the clinical controversies in classification. Previous work (41) suggests that substantial misclassification (on the order of 15 to $30 \%$, depending on whether it is uni- or bidirectional) is required to introduce false levels of genetic correlation. We found that the observed levels of correlation are unlikely to appear in the absence of underlying genetic correlation (table S6), as it is apparent that a very high degree of misclassification (up to 79\%) would be required to produce the observed correlations in the absence of any true genetic correlation and that reasonably expected misclassification would have limited impact on the observed $r_{\mathrm{g}}$ (fig. S8). Therefore, these results suggest true sharing of a substantial fraction of the common variant genetic architecture among psychiatric disorders as well as between behavioral-cognitive measures and brain disorders. We also performed large-scale simulations to explore the effect of sample size, polygenicity, and degree of correlation on power to detect significant correlations. First, we established that the observed heritability of the simulated misclassified traits in the UK Biobank data behaves as would be theoretically expected (fig. S9A) and that the effects on observed correlation (fig. S9, B and C) are in line with the estimates from family data (41). Reasonably low levels of misclassification or changes to the exact level of heritability appear unlikely to induce significant correlations, as observed in the power analysis (fig. S10), though a lower observed heritability caused by substantial misclassification (fig. S9A) will decrease the power to estimate true genetic overlap.
The high degree of genetic correlation among the psychiatric disorders adds further evidence that current clinical diagnostics do not reflect specific genetic etiology for these disorders and that genetic risk factors for psychiatric disorders do not respect clinical diagnostic boundaries. Rather, this finding suggests a more interconnected genetic etiology, in contrast to that of neurological disorders, and underscores the need to refine psychiatric diagnostics. This study may provide important "scaffolding" to support a framework for investigating mental disorders, incorporating many levels of information to understand basic dimensions of brain function.

The observed positive genetic correlations are consistent with a few hypothetical scenarios. For example, this observation may reflect the existence of some portion of common genetic risk factors conferring risks for multiple psychiatric disorders and where other distinct additional factors, both genetic and nongenetic, contribute to the eventual clinical presentation. The presreflect the phenotypic overlap between any two disorders; for example, the sharing between schizophrenia and ADHD might reflect underlying difficulties in executive functioning, which are well-established in both disorders (42), and that the shared risk arises from a partial capture of its shared genetic component. Similarly, we might speculate that a shared mechanism underlying cognitive biases may extend from overvalued ideas to delusions (ranging from anorexia nervosa and OCD to schizophrenia), and that this heritable intermediate trait confers pleiotropic risk to multiple outcomes. This kind of latent variable could give rise to the observed genetic ence of significant genetic correlation may also correlation between disorders, owing to the shared portion of variation affecting that variable. Though a combination of these is likely, more genome-wide significant loci are needed to evaluate these overlaps at the locus level.

Conversely, the low correlations observed across neurological disorders suggest that the current classification reflects relatively specific genetic etiologies, although the limited sample size for some of these disorders and the lack of inclusion of disorders conceived as "circuit-based" (e.g., restless legs syndrome, sleep disorders, and possibly essential tremor) constrain the full generalizability of this conclusion. On the basis of our observations, degenerative disorders (such as Alzheimer's and Parkinson's diseases) would therefore not be expected to share their polygenic risk profiles with a neuroimmunological disorder (such as MS) or neurovascular disorder (such as ischemic stroke). Similarly, we see limited evidence for the reported comorbidity between migraine with aura and ischemic stroke (43) $\left(r_{\mathrm{g}}=0.29, P=0.099\right)$; however, the standard errors of this comparison are too high to draw strong conclusions. At the disorder subtype level, migraine with and without aura $\left(r_{\mathrm{g}}=0.48, P=1.79 \times 10^{-5}\right)$ show substantial genetic correlation, whereas focal and generalized epilepsy $\left(r_{\mathrm{g}}=0.16, P=0.388\right)$ show much less.

The few significant correlations across neurology and psychiatry-namely, between migraine and ADHD, MDD, and TS-suggest modest shared etiological overlap across the neurology-psychiatry distinction. The comorbidity of migraine with MDD, $\mathrm{TS}$, and ADHD has been previously reported in epidemiological studies (44-47), whereas the previously reported comorbidity between migraine and bipolar disorder seen in epidemiological studies (48) was not reflected in our estimate of genetic correlation $\left(r_{\mathrm{g}}=-0.03, P=0.406\right)$.

Several phenotypes show only very low-level correlations with any of the other disorders and phenotypes that we studied, despite large sample size and robust evidence for heritability, which suggests that their common variant genetic risk may largely be unique. Alzheimer's disease, Parkinson's disease, and MS show extremely limited sharing with the other phenotypes and with each other. Neuroinflammation has been implicated in the pathophysiology of each of these conditions (49-51), as it has for migraine (52) and many psychiatric conditions, including schizophrenia (53), but no considerable shared heritability was observed with either of those conditions nor with Crohn's disease, nor did we observe enrichment for immune-related tissues in the functional partitioning (fig. S7) as observed for Crohn's disease. Although this does not preclude the sharing of individual neuroinflammatory mechanisms in these disorders, the large-scale lack of shared common variant genetic influences supports the distinctiveness of disorder etiology. Further, we observed significant enrichment of heritability for immunological cells and tissues in MS only, showing that inflammation-specific regulatory marks in the genome do not show overall enrichment for common variant risk for either Alzheimer's or Parkinson's diseases [though this does not preclude 


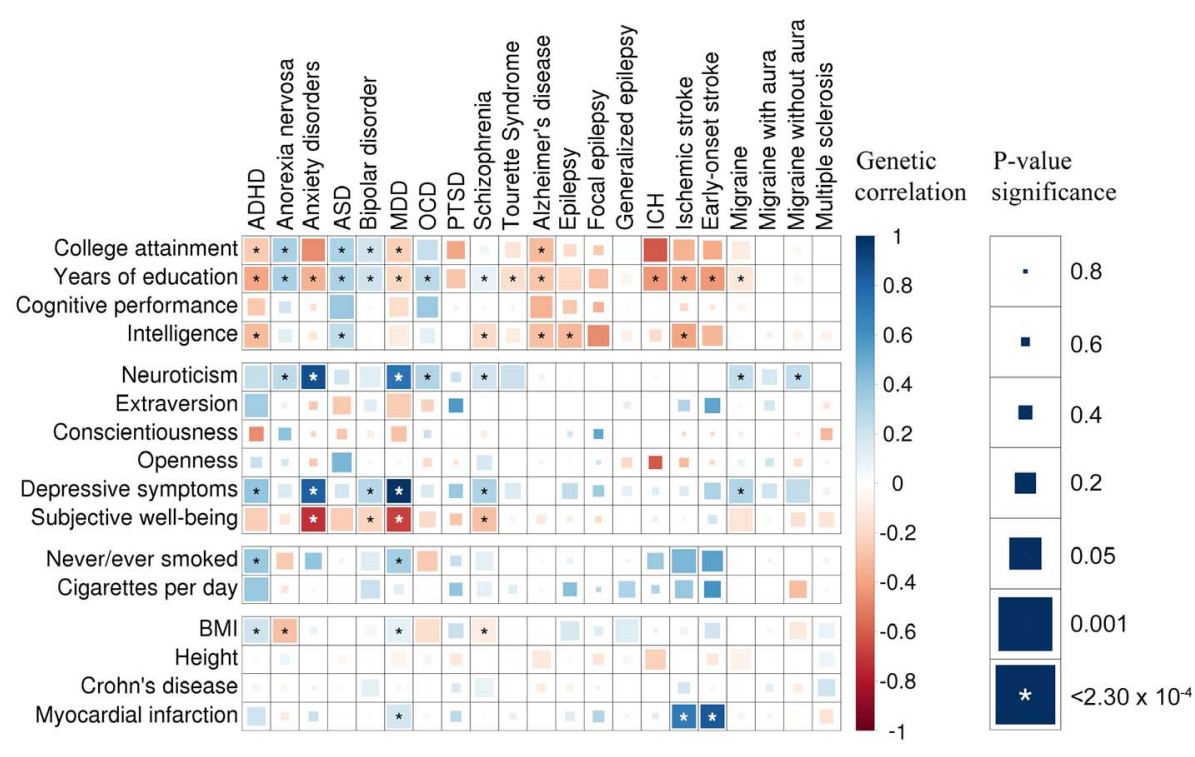

Fig. 4. Genetic correlations across brain disorders and behavioral-cognitive phenotypes. The color of each box indicates the magnitude of the correlation, and the size of the box indicates its significance (LDSC), with significant correlations filling each square completely. Asterisks indicate genetic correlations that are significantly different from zero after Bonferroni correction.

the effects of specific, not particularly polygenic neuroinflammatory mechanisms (54)]. Among psychiatric disorders, ASD and TS showed a similar absence of correlation with other disorders, although this may reflect small sample sizes.

Analysis of the Big Five personality measures suggest that the current sample sizes may be large enough for correlation testing. Neuroticism, which has by far the largest sample size, shows several significant correlations. Most significant of these was to $\operatorname{MDD}\left(r_{\mathrm{g}}=0.737, P=5.04 \times 10^{-96}\right)$, providing evidence for the link between these phenotypes, as reported for polygenic risk scores (55) and twin studies $(56,57)$; as well as other psychiatric disorders (Fig. 4 and table S7B). The correlation between MDD and anxiety disorders, with a similar pattern of correlation and the dimensional measures of depressive symptoms, subjective well-being, and neuroticism suggests that they all tag a similar underlying etiology. The significant correlation between coronary artery disease and MDD supports the link between MDD and $\mathrm{CAD}(58)$, and the observed correlation between ADHD and smoking initiation $\left(r_{\mathrm{g}}=0.374\right.$, $\left.P=3.15 \times 10^{-6}\right)$ is consistent with the epidemiological evidence of overlap (59) and findings from twin studies (60).

For the neurological disorders, five (Alzheimer's disease, intracerebral hemorrhage, ischemic and early onset stroke, and migraine) showed significant negative genetic correlation to the cognitive measures, whereas two (epilepsy and focal epilepsy) showed moderate negative genetic correlation (fig. S5). For Alzheimer's disease, poor cognitive performance in early life has been linked to increased risk for developing the disorder (61), but to our knowledge no such connection has been reported for other phenotypes.
Among the psychiatric disorders, ADHD, anxiety disorders, and MDD show a significant negative correlation to cognitive and education attainment measures, whereas the remaining five of the eight psychiatric disorders (anorexia nervosa, ASD, bipolar disorder, OCD, and schizophrenia) showed significant positive genetic correlation with one or more cognitive measures. These results suggest the existence of a link between cognitive both psychiatric and neurological brain disorders. The basis of the genetic correlations between education, cognition, and brain disorders may have a variety of root causes, including indexing performance differences on the basis of behavioral dysregulation (e.g., ADHD relating to attentional problems during cognitive tests), or may reflect ascertainment biases in certain disorders conditional on impaired cognition (e.g., individuals with lower cognitive reserve being more rapidly identified for Alzheimer's disease), but the results could also suggest a direct link between the underlying etiologies.

BMI shows significant positive genetic correlation to $\mathrm{ADHD}$, consistent with a meta-analysis linking ADHD to obesity (62), and negative genetic correlation with anorexia nervosa, OCD, and schizophrenia. This is consistent with evidence for enrichment of BMI heritability in CNS tissues (26) that suggest neuronal involvement (63); this may also provide a partial genetic explanation for lower BMI in anorexia nervosa patients even after recovery (64). Given that no strong correlations were observed between BMI and any of the neurological phenotypes, BMI's brain-specific genetic architecture may be more closely related to behavioral phenotypes. Ischemic stroke and BMI show surprisingly little genetic correlation in this performance in early life and the genetic risk for analysis $\left(r_{\mathrm{g}}=0.07, P=0.26\right)$, suggesting that although BMI is a risk factor for stroke (65), there is little evidence for shared common genetic effects. These analyses also suggest that the reported reduced rates of cardiovascular disease in individuals with histories of anorexia nervosa $(66,67)$ are more likely due to BMI-related secondary effects. The limited evidence of genetic correlation of anorexia nervosa with intracerebral hemorrhage, ischemic stroke, early onset stroke, and coronary artery disease suggests that any lower cardiovascular mortality is more likely due to direct BMI-related effects rather than to genetic risk variants.

The genetic correlation results presented here indicate that the clinical boundaries for the studied psychiatric phenotypes do not reflect distinct underlying pathogenic processes. This suggests that genetically informed analyses may provide a basis for restructuring of psychiatric nosology, consistent with twin- and family-based results. In contrast, neurological disorders show greater genetic specificity, and although it is important to emphasize that while some brain disorders are underrepresented here, our results demonstrate the limited evidence for widespread common genetic risk sharing between psychiatric and neurological disorders. However, we provide strong evidence that both psychiatric and neurological disorders show robust correlations with cognitive and personality measures, indicating avenues for follow-up studies. Further analysis is needed to evaluate whether overlapping genetic contributions to psychiatric pathology may influence treatment choices. Ultimately, such developments are promising steps toward reducing diagnostic heterogeneity and eventually improving the diagnostics and treatment of psychiatric disorders.

\section{Materials and methods summary}

We collected GWAS meta-analysis summary statistics for 25 brain disorders and 17 other phenotypes from various consortia and, where necessary, generated new, non-sex-stratified European cohortonly versions of the meta-analyses (25). All datasets underwent uniform quality control (25). For each trait, by using the LDSC framework (24), the total additive common SNP heritability present in the summary statistics $\left(h^{2} g\right)$ was estimated by regressing the association $\chi^{2}$ statistic of a SNP against the total amount of common genetic variation tagged by that SNP, for all SNPs. Genetic correlations $\left(r_{\mathrm{g}}\right.$; i.e., the genome-wide average shared genetic risk) for pairs of phenotypes were estimated by regressing the product of $z$-scores, rather than the $\chi^{2}$ statistic, for each phenotype and for each SNP. Significance was assessed by Bonferroni multiple testing correction via estimating the number of independent brain disorder phenotypes via matrix decomposition (25). Functional and partitioning analyses for the GWAS datasets were also performed using LDSC regression. Power analyses and simulation work to aid in interpretation of the results were conducted using genotype data from the UK Biobank resource (25). 


\section{REFERENCES AND NOTES}

1. J. B. Martin, The integration of neurology, psychiatry, and neuroscience in the 21st century. Am. J. Psychiatry 159, 695-704 (2002). doi: 10.1176/appi.ajp.159.5.695; pmid: 11986119

2. J. W. Smoller, Disorders and borders: Psychiatric genetics and nosology. Am. J. Med. Genet. B. Neuropsychiatr. Genet. 162 , 559-578 (2013). doi: 10.1002/ajmg.b.32174; pmid: 24132891

3. T. R. Insel, P. S. Wang, Rethinking mental illness. JAMA 303, 1970-1971 (2010). doi: 10.1001/jama.2010.555; pmid: 20483974

4. T. J. Polderman et al., Meta-analysis of the heritability of human traits based on fifty years of twin studies. Nat. Genet. 47, 702-709 (2015). doi: 10.1038/ng.3285; pmid: 25985137

5. K. S. Kendler, C. A. Prescott, J. Myers, M. C. Neale, The structure of genetic and environmental risk factors for common psychiatric and substance use disorders in men and women. Arch. Gen. Psychiatry 60, 929-937 (2003). doi: 10.1001/archpsyc.60.9.929; pmid: 12963675

6. R. Jensen, L. J. Stovner, Epidemiology and comorbidity of headache. Lancet Neurol. 7, 354-361 (2008). doi: 10.1016/ S1474-4422(08)70062-0; pmid: 18339350

7. J. Nuyen et al., Comorbidity was associated with neurologic and psychiatric diseases: A general practice-based controlled study. J. Clin. Epidemiol. 59, 1274-1284 (2006). doi: 10.1016/ j.jclinepi.2006.01.005; pmid: 17098570

8. R. M. Hirschfeld et al., Screening for bipolar disorder in the community. J. Clin. Psychiatry 64, 53-59 (2003). doi: 10.4088/JCP.v64n0111; pmid: 12590624

9. A. Pan, Q. Sun, O. I. Okereke, K. M. Rexrode, F. B. Hu, Depression and risk of stroke morbidity and mortality: A meta-analysis and systematic review. JAMA 306, 1241-1249 (2011). doi: 10.1001/jama.2011.1282; pmid: 21934057

10. A. Lo-Castro, P. Curatolo, Epilepsy associated with autism and attention deficit hyperactivity disorder: Is there a genetic link? Brain Dev. 36, 185-193 (2014). doi: 10.1016/ j.braindev.2013.04.013; pmid: 23726375

11. E. N. Bertelsen, J. T. Larsen, L. Petersen, J. Christensen, S. Dalsgaard, Childhood Epilepsy, Febrile Seizures, and Subsequent Risk of ADHD. Pediatrics 138, e20154654 (2016). doi: 10.1542/peds.2015-4654; pmid: 27412639

12. C. G. de Kovel et al., Recurrent microdeletions at $15 q 11.2$ and $16 \mathrm{p} 13.11$ predispose to idiopathic generalized epilepsies Brain 133, 23-32 (2010). doi: 10.1093/brain/awp262; pmid: 19843651

13. T. D. Graves, M. G. Hanna, Neurological channelopathies. Postgrad. Med. J. 81, 20-32 (2005). doi: 10.1136/ pgmj.2004.022012; pmid: 15640425

14. J. Haan, G. M. Terwindt, A. M. van den Maagdenberg, A. H. Stam, M. D. Ferrari, A review of the genetic relation between migraine and epilepsy. Cephalalgia 28, 105-113 (2008). pmid: 18197881

15. S. Debette et al., Common variation in PHACTR1 is associated with susceptibility to cervical artery dissection. Nat. Genet. 47, 78-83 (2015). doi: 10.1038/ng.3154; pmid: 25420145

16. S. M. Purcell et al., Common polygenic variation contributes to risk of schizophrenia and bipolar disorder. Nature 460 748-752 (2009). pmid: 19571811

17. Cross-Disorder Group of the Psychiatric Genomics Consortium, Genetic relationship between five psychiatric disorders estimated from genome-wide SNPs. Nat. Genet. 45, 984-994 (2013). doi: 10.1038/ng.2711; pmid: 23933821

18. J. C. Lambert et al., Meta-analysis of 74,046 individuals identifies 11 new susceptibility loci for Alzheimer's disease. Nat. Genet. 45, 1452-1458 (2013). doi: 10.1038/ng.2802; pmid: 24162737

19. T. W. Mühleisen et al., Genome-wide association study reveals two new risk loci for bipolar disorder. Nat. Commun. 5, 3339 (2014). doi: 10.1038/ncomms4339; pmid: 24618891

20. V. Anttila et al., Genome-wide meta-analysis identifies new susceptibility loci for migraine. Nat. Genet. 45, 912-917 (2013). doi: 10.1038/ng.2676; pmid: 23793025

21. M. A. Nalls et al., Large-scale meta-analysis of genome-wide association data identifies six new risk loci for Parkinson's disease. Nat. Genet. 46, 989-993 (2014). doi: 10.1038/ ng.3043; pmid: 25064009

22. Schizophrenia Working Group of the Psychiatric Genomics Consortium, Biological insights from 108 schizophreniaassociated genetic loci. Nature 511, 421-427 (2014) doi: 10.1038/nature13595; pmid: 25056061

23. N. Solovieff, C. Cotsapas, P. H. Lee, S. M. Purcell, J. W. Smoller, Pleiotropy in complex traits: Challenges and strategies. Nat. Rev. Genet. 14, 483-495 (2013). doi: 10.1038/nrg3461 pmid: 23752797
24. B. Bulik-Sullivan et al., An atlas of genetic correlations acros human diseases and traits. Nat. Genet. 47, 1236-1241 (2015). doi: 10.1038/ng.3406; pmid: 26414676

25. See materials and methods and other supplementary materials.

26. H. K. Finucane et al., Partitioning heritability by functional annotation using genome-wide association summary statistics. Nat. Genet. 47, 1228-1235 (2015). doi: 10.1038/ng.3404; pmid: 26414678

27. M. H. de Moor et al., Meta-analysis of genome-wide association studies for personality. Mol. Psychiatry 17, 337-349 (2012) doi: 10.1038/mp.2010.128; pmid: 21173776

28. R. A. Power, M. Pluess, Heritability estimates of the Big Five personality traits based on common genetic variants. Transl. Psychiatry 5, e604 (2015). doi: 10.1038/tp.2015.96; pmid: 26171985

29. C. M. Haworth et al., The heritability of general cognitive ability increases linearly from childhood to young adulthood. Mol. Psychiatry 15, 1112-1120 (2010). doi: 10.1038/ mp.2009.55; pmid: 19488046

30. I. J. Deary et al., Genetic contributions to stability and change in intelligence from childhood to old age. Nature 482, 212-215 (2012). doi: 10.1038/nature10781; pmid: 22258510

31. S. De Rubeis et al., Synaptic, transcriptional and chromatin genes disrupted in autism. Nature 515, 209-215 (2014). doi: 10.1038/nature13772; pmid: 25363760

32. S. H. Lee et al., Estimation and partitioning of polygenic variation captured by common SNPs for Alzheimer's disease multiple sclerosis and endometriosis. Hum. Mol. Genet. 22. 832-841 (2013). doi: 10.1093/hmg/dds491; pmid: 23193196

33. A. Okbay et al., Genome-wide association study identifies 74 loci associated with educational attainment. Nature $\mathbf{5 3 3}$ 539-542 (2016). doi: 10.1038/nature17671; pmid: 27225129

34. D. J. Smith et al., Genome-wide analysis of over 106000 individuals identifies 9 neuroticism-associated loci. Mol. Psychiatry 21, 749-757 (2016). doi: 10.1038/mp.2016.49; pmid: 27067015

35. P. F. Buckley, B. J. Miller, D. S. Lehrer, D. J. Castle, Psychiatric comorbidities and schizophrenia. Schizophr. Bull. 35, 383-402 (2009). doi: 10.1093/schbul/sbn135; pmid: 19011234

36. C. G. Lyketsos et al., Mental and behavioral disturbances in dementia: Findings from the Cache County Study on Memory in Aging. Am. J. Psychiatry 157, 708-714 (2000) doi: 10.1176/appi.ajp.157.5.708; pmid: 10784462

37. R. Kendell, A. Jablensky, Distinguishing between the validity and utility of psychiatric diagnoses. Am. J. Psychiatry 160 , 4-12 (2003). doi: 10.1176/appi.ajp.160.1.4; pmid: 12505793

38. A. S. Cristino et al., Neurodevelopmental and neuropsychiatric disorders represent an interconnected molecular system. Mol. Psychiatry 19, 294-301 (2014). doi: 10.1038/mp.2013.16; pmid: 23439483

39. D. A. Regier et al., Limitations of diagnostic criteria and assessment instruments for mental disorders. Implications for research and policy. Arch. Gen. Psychiatry 55, 109-115 (1998). doi: 10.1001/archpsyc.55.2.109; pmid: 9477922

40. T. M. Laursen, E. Agerbo, C. B. Pedersen, Bipolar disorder schizoaffective disorder, and schizophrenia overlap: A new comorbidity index. J. Clin. Psychiatry 70, 1432-1438 (2009). doi: 10.4088/JCP.08m04807; pmid: 19538905

41. N. R. Wray, S. H. Lee, K. S. Kendler, Impact of diagnostic misclassification on estimation of genetic correlations using genome-wide genotypes. Eur. J. Hum. Genet. 20, 668-674 (2012). doi: 10.1038/ejhg.2011.257; pmid: 2225852

42. E. G. Willcutt, A. E. Doyle, J. T. Nigg, S. V. Faraone, B. F. Pennington, Validity of the executive function theory of attention-deficit/hyperactivity disorder: A meta-analytic review. Biol. Psychiatry 57, 1336-1346 (2005). doi: 10.1016/ j.biopsych.2005.02.006; pmid: 15950006

43. J. T. Spector et al., Migraine headache and ischemic stroke risk: An updated meta-analysis. Am. J. Med. 123 612-624 (2010). doi: 10.1016/j.amjmed.2009.12.021; pmid: 20493462

44. O. B. Fasmer, A. Halmøy, K. J. Oedegaard, J. Haavik, Adult attention deficit hyperactivity disorder is associated with migraine headaches. Eur. Arch. Psychiatry Clin. Neurosci. 261. 595-602 (2011). doi: 10.1007/s00406-011-0203-9; pmid: 21394551

45. N. Breslau, R. B. Lipton, W. F. Stewart, L. R. Schultz, K. M. Welch, Comorbidity of migraine and depression: Investigating potential etiology and prognosis. Neurology 60 1308-1312 (2003). doi: 10.1212/01. WNL.0000058907.41080.54; pmid: 12707434

46. K. R. Merikangas, J. Angst, H. Isler, Migraine and psychopathology. Results of the Zurich cohort study of young adults. Arch. Gen.
Psychiatry 47, 849-853 (1990). doi: 10.1001/archpsyc.1990. 01810210057008; pmid: 2393343

47. G. Barabas, W. S. Matthews, M. Ferrari, Tourette's syndrome and migraine. Arch. Neurol. 41, 871-872 (1984). doi: 10.1001/ archneur.1984.04050190077018; pmid: 6589980

48. R. S. Mclntyre et al., The prevalence and impact of migraine headache in bipolar disorder: Results from the Canadian Community Health Survey. Headache 46, 973-982 (2006). doi: 10.1111/j.1526-4610.2006.00469.x; pmid: 16732843

49. M. T. Heneka et al., Neuroinflammation in Alzheimer's disease. Lancet Neurol. 14, 388-405 (2015). doi: 10.1016/S14744422(15)70016-5; pmid: 25792098

50. E. C. Hirsch, S. Hunot, Neuroinflammation in Parkinson's disease: A target for neuroprotection? Lancet Neurol. 8 , 382-397 (2009). doi: 10.1016/S1474-4422(09)70062-6; pmid: 19296921

51. E. M. Frohman, M. K. Racke, C. S. Raine, Multiple sclerosis-the plaque and its pathogenesis. N. Engl. J. Med. 354, 942-955 (2006). doi: 10.1056/NEJMra052130; pmid: 16510748

52. C. Waeber, M. A. Moskowitz, Migraine as an inflammatory disorder. Neurology 64 (Suppl 2), S9-S15 (2005). doi: 10.1212/ WNL.64.10_suppl_2.S9; pmid: 15911785

53. J. Steiner et al., Increased prevalence of diverse N-methyl-D aspartate glutamate receptor antibodies in patients with an initial diagnosis of schizophrenia: Specific relevance of IgG NRla antibodies for distinction from N-methyl-D-aspartate glutamate receptor encephalitis. JAMA Psychiatry 70 271-278 (2013). doi: 10.1001/2013.jamapsychiatry.86; pmid: 23344076

54. L. Jones et al., Convergent genetic and expression data implicate immunity in Alzheimer's disease. Alzheimers Dement. 11, 658-671 (2015). doi: 10.1016/j.jalz.2014.05.1757; pmid: 25533204

55. M. H. M. de Moor et al., Meta-analysis of Genome-wide Association Studies for Neuroticism, and the Polygenic Association With Major Depressive Disorder. JAMA Psychiatry 72, 642-650 (2015). doi: 10.1001/jamapsychiatry.2015.0554; pmid: 25993607

56. K. S. Kendler, M. Gatz, C. O. Gardner, N. L. Pedersen, Personality and major depression: A Swedish longitudinal, population-based twin study. Arch. Gen. Psychiatry 63 1113-1120 (2006). doi: 10.1001/archpsyc.63.10.1113; pmid: 17015813

57. R. E. Ørstavik, K. S. Kendler, N. Czajkowski, K. Tambs, T. Reichborn-Kjennerud, The relationship between depressive personality disorder and major depressive disorder: A populationbased twin study. Am. J. Psychiatry 164, 1866-1872 (2007). doi: 10.1176/appi.ajp.2007.07010045; pmid: 18056242

58. H. Hemingway, M. Marmot, Evidence based cardiology: Psychosocial factors in the aetiology and prognosis of coronary heart disease. Systematic review of prospective cohort studies. BMJ 318, 1460-1467 (1999). doi: 10.1136/ bmi.318.7196.1460; pmid: 10346775

59. F. J. McClernon, S. H. Kollins, ADHD and smoking: From genes to brain to behavior. Ann. N. Y. Acad. Sci. 1141, 131-147 (2008). doi: 10.1196/annals.1441.016; pmid: 18991955

60. T. Korhonen et al., Externalizing behaviors and cigarette smoking as predictors for use of illicit drugs: A longitudinal study among Finnish adolescent twins. Twin Res. Hum. Genet. 13, 550-558 (2010). doi: 10.1375/twin.13.6.550; pmid: 21142931

61. D. A. Snowdon et al., Linguistic ability in early life and cognitive function and Alzheimer's disease in late life. Findings from the Nun Study. JAMA 275, 528-532 (1996). doi: 10.1001/ jama.1996.03530310034029; pmid: 8606473

62. S. Cortese et al., Association Between ADHD and Obesity: A Systematic Review and Meta-Analysis. Am. J. Psychiatry 173 34-43 (2016). doi: 10.1176/appi.ajp.2015.15020266; pmid: 26315982

63. A. E. Locke et al., Genetic studies of body mass index yield new insights for obesity biology. Nature 518, 197-206 (2015) doi: 10.1038/nature14177; pmid: 25673413

64. L. Mustelin et al., Long-term outcome in anorexia nervosa in the community. Int. J. Eat. Disord. 48, 851-859 (2015) doi: 10.1002/eat.22415; pmid: 26059099

65. T. Kurth et al., Prospective study of body mass index and risk of stroke in apparently healthy women. Circulation 111 1992-1998 (2005). doi: 10.1161/01.CIR.0000161822.83163.B6; pmid: 15837954

66. S. R. Korndörfer et al., Long-term survival of patients with anorexia nervosa: A population-based study in Rochester Minn. Mayo Clin. Proc. 78, 278-284 (2003). doi: 10.4065/ 78.3.278; pmid: 12630579 
67. P. F. Sullivan, Discrepant results regarding long-term survival of patients with anorexia nervosa? Mayo Clin. Proc. 78, 273-274 (2003). doi: 10.4065/ 78.3.273; pmid: 12630577

\section{ACKNOWLEDGMENTS}

We thank the members of the Neale and Daly laboratories for helpful discussions; R. Hoskins, J. Wessman, and J. Martin for comments on the manuscript; M. Whittall for inspiration; S. Knemeyer for help with the summary figure; C. Hammond for organizational assistance; and the patients and participants of the respective consortia for their participation. Data on coronary artery disease have been contributed by CARDIoGRAMplusC4D investigators and have been downloaded from www.cardiogramplusc4d.org. matSpD (Matrix Spectral Decomposition method) is available at neurogenetics.qimrberghofer.edu.au/matSpD/. This research was conducted using the UK Biobank resource (application 18597).

Funding: This work was supported by grants 1R01MH10764901 and 5U01MH09443203 from the National Institute of Mental Health, as well as the Orion Farmos Research Foundation (V.A.) and the Fannie and John Hertz Foundation (H.K.F.)

Consortium specific funding is detailed in the supplementary materials ("Study-specific acknowledgments"). Author contributions: V.A., A.C., and B.M.N. conceived of and coordinated the study; V.A., B.B.S., H.K.F., R.W., and P.T. contributed methodology; B.B.S. and H.K.F. contributed software; V.A. and B.M.N. conducted the statistical analysis; B.M.N. obtained funding and provided resources; R.W., J.B., L.D., V.E.-P., G.F. P.G., R.M., N.P., S.R., Z.W., and D.Y. were responsible for curation of disorder-specific data; P.H.L. and C.C. helped with data interpretation; V.A. and B.M.N. wrote the original draft, and all authors contributed to review and editing; V.A. provided the visualization; G.B., C.B., M.Daly, M.Dichgans, S.V.F., R.G., P.H., K.K., B.K., C.A.M., A.P., J.S., P.S., J.W., N.W., C.C., A.P., J.S., P.S., J.R., A.C., and B.M.N. provided supervision and project administration; and the remaining authors contributed disorder-specific sample collection and/or analysis. Consortium-specific personnel lists can be found in the supplementary materials. Competing interests: The authors declare no competing interests. Data and materials availability: Data sources for the GWAS summary statistics used in the study and their availability, as well as the study-specific acknowledgments, are provided in the supplementary materials (table S13 and supplementary text, respectively).

\section{The Brainstorm Consortium}

Verneri Anttila ${ }^{12,3 *}$, Brendan Bullik-Sullivann, ${ }^{13}$, Hilary K. Finucane $e^{2,3,4,5}$ Raymond K. Walters $s^{1,2,3}$, Jose Bras, ${ }^{6,7}$, Laramie Duncan ${ }^{1,2,3,8}$ Valentina Escott-Price ${ }^{9,10}$, Guido J. Falcone ${ }^{11,12,13}$, Padhraig Gormley 1,2,3,11, Rainer Malik ${ }^{14}$, Nikolaos A. Patsopoulos, ${ }^{3,15}$, Stephan Ripke, 1,2,16, Zhi Wei ${ }^{17}$, Dongmei Yu ${ }^{2,11}$, Phil H. Lee ${ }^{2,11}$, Patrick Turley ${ }^{1,3}$, Benjamin Grenier-Boley ${ }^{18,19,20}$, Vincent Chouraki ${ }^{18,19,20,21}$ Yoichiro Kamatani $i^{22,23}$, Claudine Berr ${ }^{24,25,26}$, Luc Letenneur ${ }^{27,28}$ Didier Hannequin ${ }^{29,30}$, Philippe Amouyel ${ }^{18,19,20,21}$, Anne Boland ${ }^{31}$ Jean-François Deleuze ${ }^{31}$, Emmanuelle Duron ${ }^{32,33}$, Badri N. Vardarajan ${ }^{34}$ Christiane Reitz $^{35}$, Alison M. Goate ${ }^{36}$, Matthew J. Huentelman ${ }^{37}$, M. Ilyas Kamboh ${ }^{38}$, Eric B. Larson ${ }^{39,40}$, Ekaterina Rogaeva $a^{41}$. Peter St George-Hyslop ${ }^{41,42}$, Hakon Hakonarson ${ }^{43,44,45}$, Walter A. Kukull ${ }^{46}$, Lindsay A. Farrer ${ }^{47}$, Lisa L. Barnes ${ }^{48,49,50}$, Thomas G. Beach ${ }^{51}$, F. Yesim Demirci ${ }^{38}$, Elizabeth Head ${ }^{52}$, Christine M. Hulette ${ }^{53}$, Gregory A. Jicha ${ }^{54}$, John S.K. Kauwe ${ }^{55}$, Jeffrey A. Kaye ${ }^{56}$, James B. Leverenz ${ }^{57}$, Allan I. Levey ${ }^{58}$, Andrew P. Lieberman ${ }^{59}$, Vernon S. Pankratz ${ }^{60}$, Wayne W. Poon ${ }^{61}$, Joseph F. Quinn ${ }^{62,63}$ Andrew J. Saykin ${ }^{64}$, Lon S. Schneider ${ }^{65}$, Amanda G. Smith ${ }^{66}$, Joshua A. Sonnen ${ }^{67,68}$, Robert A. Stern ${ }^{69}$, Vivianna M. Van Deerlin ${ }^{70}$ Linda J. Van Eldik ${ }^{52}$, Denise Harold ${ }^{71}$, Giancarlo Russo ${ }^{72}$, David C. Rubinsztein ${ }^{73,74}$, Anthony Bayer ${ }^{75}$, Magda Tsolaki ${ }^{76,77}$, Petra Proitsi ${ }^{78}$, Nick C. Fox ${ }^{79,6}$, Harald Hampe ${ }^{80,81,82,83}$, Michael J. Owen ${ }^{84,85}$, Simon Mead ${ }^{86}$, Peter Passmore ${ }^{87}$, Kevin Morgan ${ }^{88}$, Markus M. Nöthen ${ }^{89,90}$. Martin Rossor ${ }^{91}$. Michelle K. Lupton ${ }^{92,93}$ Per Hoffmann ${ }^{89,90,94,95}$, Johannes Kornhuber ${ }^{96}$, Brian Lawlor ${ }^{97}$ Andrew McQuillin ${ }^{98}$, Ammar Al-Chalabi ${ }^{99,100}$, Joshua C. Bis ${ }^{101}$ Agustin Ruiz ${ }^{102,103}$, Mercè Boada ${ }^{102}$, Sudha Seshadri ${ }^{104,105,106}$ ' Alexa Beiser ${ }^{107,108,106}$, Kenneth Rice ${ }^{109}$, Sven J. van der Lee ${ }^{110}$, Philip L. De Jager ${ }^{111}$, Daniel H. Geschwind ${ }^{12,113,114}$, Matthias Riemenschneider ${ }^{115}$, Steffi Riedel-Heller ${ }^{116}$, Jerome I. Rotter ${ }^{117}$ Gerhard Ransmayr ${ }^{118}$, Bradley T. Hyman ${ }^{12,13}$, Carlos Cruchaga ${ }^{120}$, Montserrat Alegret ${ }^{102}$, Bendik Winsvold ${ }^{121,122}$, Priit Palta ${ }^{123,124}$, Kai-How Farh ${ }^{125,3}$, Ester Cuenca-Leon ${ }^{11,3,2}$, Nicholas Furlotte ${ }^{126}$, Tobias Kurth ${ }^{127}$, Lannie Ligthart ${ }^{128}$, Gisela M.Terwindt ${ }^{129}$ Tobias Freilinger ${ }^{130,131}$, Caroline Ran ${ }^{132}$, Scott D.Gordon ${ }^{92}$, Guntram Borck ${ }^{133}$, Hieab H.H. Adams ${ }^{110,134}$, Terho Lehtimäki ${ }^{135}$ Juho Wedenoja ${ }^{136,137}$, Julie E. Buring ${ }^{138}$, Markus Schürks ${ }^{139}$ Maria Hrafnsdottir ${ }^{140}$, Jouke-Jan Hottenga ${ }^{128,141}$, Brenda Penninx ${ }^{142}$
Ville Artto $^{143}$, Mari Kaunisto ${ }^{123}$, Salli Vepsäläinen ${ }^{143}$ Nicholas G. Martin ${ }^{92}$, Grant W. Montgomery ${ }^{92,144}$, Mitja I. Kurki ${ }^{1,3,11,123}$, Eija Hämäläinen ${ }^{123}$, Hailiang Huang ${ }^{1,2,145}$, Jie Huang ${ }^{146,147}$, Cynthia Sandor $^{148}$, Caleb Webber ${ }^{148,10}$, Bertram Muller-Myhsok ${ }^{149,150,151}$, Stefan Schreiber ${ }^{152,153}$, Veikko Salomaa ${ }^{154}$, Elizabeth Loehrer ${ }^{155}$, Hartmut Göbel ${ }^{156}$ Alfons Macaya ${ }^{157}$ Patricia Pozo-Rosich ${ }^{158,159}{ }^{\text {' }}$ Thomas Hansen ${ }^{160,161}$, Thomas Werge ${ }^{161,162,163}$, Jaakko Kaprio ${ }^{123,137}$ Andres Metspalu ${ }^{124}$, Christian Kubisch ${ }^{164}$, Michel D. Ferrari ${ }^{129}$, Andrea C. Belin ${ }^{132}$, Arn M. J. M. van den Maagdenberg ${ }^{166,129}$, John-Anker Zwart ${ }^{167}$, Dorret Boomsma ${ }^{168.128}$, Nicholas Eriksson ${ }^{126}$, Jes Olesen ${ }^{160}$, Daniel I. Chasmann ${ }^{169,13}$, Dale R. Nyholt ${ }^{170}$, Andreja Avbersek ${ }^{171}$, Larry Baum ${ }^{172}$, Samuel Berkovic ${ }^{173}$, Jonathan Bradfield ${ }^{174}$, Russell Buono ${ }^{175,176,177}$, Claudia B. Catarino ${ }^{171,178}$ Patrick Cossette ${ }^{179}$, Peter De Jonghe ${ }^{180,181,182}$, Chantal Depondt ${ }^{183}$ Dennis Dlugos $^{184,185}$, Thomas N. Ferraro ${ }^{186,187}$, Jacqueline French ${ }^{188}$ Helle Hjalgrim $^{189}$, Jennifer Jamnadas-Khoda ${ }^{171,190}$, Reetta Kälviäinen ${ }^{192,193}$, Wolfram S. Kunz ${ }^{194,195}$, Holger Lerche ${ }^{131}$, Costin Leu ${ }^{196}$, Dick Lindhout ${ }^{197,198}$, Warren Lo L9,200, Daniel Lowenstein ${ }^{201}$, Mark McCormack ${ }^{202,203}$, Rikke S. Møøller ${ }^{204,205}$, Anne Molloy' ${ }^{206}$ Ping-Wing Ng ${ }^{207,208}$, Karen Oliver ${ }^{209}$, Michael Privitera ${ }^{210,211}$, Rodney Radtke ${ }^{212}$, Ann-Kathrin Ruppert ${ }^{213}$, Thomas Sander ${ }^{213}$ Steven Schachter ${ }^{214,12,13}$, Christoph Schankin ${ }^{215,216}$ Ingrid Scheffer $217,218,219$, Susanne Schoch ${ }^{220}$, Sanjay M. Sisodiyaa ${ }^{221,222}$ Philip Smith ${ }^{223}$, Michael Sperling ${ }^{224}$, Pasquale Striano ${ }^{225}$, Rainer Surges ${ }^{226.227}$, G. Neil Thomas ${ }^{228}$, Frank Visscher ${ }^{229}$, Christopher D. Whelan ${ }^{202}$, Federico Zara ${ }^{230}$, Erin L. Heinzen ${ }^{231}$ Anthony Marson ${ }^{232,233}$, Felicitas Becker ${ }^{234,235}$. Hans Stroink ${ }^{236}$ Fritz Zimprich ${ }^{237}$, Thomas Gasser ${ }^{238,239}$, Raphael Gibbs ${ }^{240}$ Peter Heutink ${ }^{239,238}$, Maria Martinez ${ }^{241,242}$, Huw R. Morris ${ }^{221}$ Manu Sharma ${ }^{243}$, Mina Ryten ${ }^{221}$, Kin Y. Mok ${ }^{7,245}$, Sara Pulit ${ }^{24,247,3}$, Steve Bevan ${ }^{248}$, Elizabeth Holliday ${ }^{249}$ John Attia ${ }^{250,251}$, Thomas Battey ${ }^{11,253}$, Giorgio Boncoraglio 254,255 Vincent Thijs ${ }^{219,256}$, Wei-Min Chen ${ }^{257}$. Braxton Mitchell| 258,259 Peter Rothwell ${ }^{260}$, Pankaj Sharma ${ }^{261,262}$, Cathie Sudlow ${ }^{263}$, Astrid Vicente ${ }^{264,265}$. Hugh Markus ${ }^{266}$, Christina Kourkoulis ${ }^{3,119,11}$ Joana Pera ${ }^{267}$, Miriam Raffeld ${ }^{3,11,119,268}$, Scott Silliman ${ }^{269}$, Vesna Boraska Perica ${ }^{270}$, Laura M. Thornton ${ }^{271}$, Laura M. Huckins ${ }^{272}$ N. William Rayner ${ }^{273,274,275}$, Cathryn M. Lewis ${ }^{276}$, Monica Gratacos ${ }^{27}$ Filip Rybakowski ${ }^{278}$, Anna Keski-Rahkonen ${ }^{279}$, Anu Raevuori ${ }^{280,279}$ James I. Hudson ${ }^{281}$, Ted Reichborn-Kjennerud ${ }^{282,283}$ Palmiero Monteleone ${ }^{284}$, Andreas Karwautz ${ }^{285}$, Katrin Mannik ${ }^{124,286}$ Jessica H. Baker ${ }^{271}$, Julie K. O'Toole ${ }^{287}$, Sara E. Trace ${ }^{288}$, Oliver S. P. Davis ${ }^{289}$, Sietske G. Helder ${ }^{290,276}$, Stefan Ehrlich ${ }^{291}$ Beate Herpertz-Dahlmann ${ }^{292}$, Unna N. Danner ${ }^{293,294}$ Annemarie A. van Elburg ${ }^{293,294}$, Maurizio Clementi ${ }^{295}$, Monica Forzan ${ }^{296}$, Elisa Docampo ${ }^{297,298}$, Jolanta Lissowska ${ }^{299}$, Joanna Hauser ${ }^{300}$, Alfonso Tortorella ${ }^{301}$, Mario Maj ${ }^{302}$, Fragiskos Gonidakis ${ }^{303}$, Konstantinos Tziouvas ${ }^{304}$, Hana Papezova ${ }^{305,306}$, Zeynep Yilmaz ${ }^{271}$, Gudrun Wagner ${ }^{307}$. Sarah Cohen-Woods ${ }^{308}$ Stefan Herms ${ }^{89,90,94,95}$, Antonio Juliài ${ }^{311}$, Raquel Rabionet ${ }^{312,313,314,315}$ Danielle M. Dick ${ }^{316}$, Samuli Ripatti ${ }^{123,137,317}$, Ole A. Andreassen ${ }^{318,319}$ ', Thomas Espeseth ${ }^{318,320,321}$. Astri J. Lundervold ${ }^{322,321}$ Vidar M. Steen ${ }^{323,324}$, Dalila Pinto ${ }^{325,326,327,328}$ Stephen W. Scherer ${ }^{330,331}$, Harald Aschauer ${ }^{332}$ Alexandra Schosser ${ }^{333,334}$, Lars Alfredsson ${ }^{335}$, Leonid Padyukov ${ }^{336}$ Katherine A. Halmi ${ }^{337}$, James Mitchell ${ }^{338,339}$, Michael Strober ${ }^{340}$, Andrew W. Bergen ${ }^{341,342}$. Walter Kaye ${ }^{343}$, Jin Peng Szatkiewicz ${ }^{271}$ Bru Cormand ${ }^{312,313,314,344}$, Josep Antoni Ramos-Quiroga ${ }^{345,346,348,347}$ Cristina Sánchez-Mora ${ }^{346,345,348}$, Marta Ribasés ${ }^{346,345,348,}$ Miguel Casas ${ }^{349,350,345,351}$, Amaia Hervas ${ }^{352}$, Maria Jesús Arranz ${ }^{353}$, Jan Haavik ${ }^{354,355}$, Tetyana Zayats ${ }^{354,1}$. Stefan Johansson ${ }^{356,324}$, Nigel Williams ${ }^{9}$, Astrid Dempfle ${ }^{357}$, Aribert Rothenberger ${ }^{358}$, Jonna Kuntsi ${ }^{359}$, Robert D. Oades ${ }^{360}$, Tobias Banaschewski ${ }^{361}$ Barbara Franke ${ }^{362,363,364}$, Jan K. Buitelaar ${ }^{365,366}$ Alejandro Arias Vasquez ${ }^{367}$, Alysa E. Doyle ${ }^{119,13}$, Andreas Reif ${ }^{368}$, Klaus-Peter Lesch ${ }^{369,370,371}$, Christine Freitag ${ }^{372}$, Olga Rivero ${ }^{371}$ Haukur Palmason ${ }^{140}$, Marcel Romanos ${ }^{373}$, Kate Langley ${ }^{374,84}$ Marcella Rietsche $\left.\right|^{375}$. Stephanie H. Witt ${ }^{375}$, Soeren Dalsgaard ${ }^{376,163,377}$, Anders D. Børglum 378,163,379,380 Irwin Waldman ${ }^{381}$, Beth Wilmot ${ }^{382}$, Nikolas Molly ${ }^{383}$ Claiton H.D. Bau ${ }^{384,385}$, Jennifer Crosbie ${ }^{386,387}$, Russell Schachar ${ }^{388,387}$, Sandra K. Loo ${ }^{389}$, James J. McGough ${ }^{390}$ Eugenio H. Grevet ${ }^{385,392}$, Sarah E. Medland ${ }^{92}$, Elise Robinson ${ }^{1,2,5}$, Lauren A. Weiss ${ }^{393,394,395}$, Elena Bacchelli ${ }^{396}$, Anthony Bailey ${ }^{397,398}$, Vanessa Bal ${ }^{393,394,395}$, Agatino Battaglia ${ }^{399}$, Catalina Betancur ${ }^{400}$ Patrick Bolton ${ }^{359,401}$, Rita Cantor ${ }^{402}$, Patrícia Celestino-Soper ${ }^{403}$, Geraldine Dawson $^{404}$, Silvia De Rubeis ${ }^{325,326,407}$ Frederico Duque ${ }^{406,405}$, Andrew Green ${ }^{408,409}$, Sabine M. Klauck ${ }^{410}$ Marion Leboyer ${ }^{411,412,413}$. Pat Levitt ${ }^{414,65}$. Elena Maestrini396, Shrikant Mane ${ }^{415,416}$, Daniel Moreno-De-Luca ${ }^{417}$, Jeremy Parr '418,419,420, Regina Regan ${ }^{409,421}$, Abraham Reichenberg ${ }^{272}$, Sven Sandin ${ }^{325,326,422}$ Jacob Vorstman ${ }^{423,424}$, Thomas Wassink ${ }^{425}$, Ellen Wijsman ${ }^{426,109}$, Edwin Cook ${ }^{427}$. Susan Santangelo ${ }^{429,430}$, Richard Delorme ${ }^{431,432}$,
Bernadette Rogé $e^{433,434,435}$, Tiago Magalhaes ${ }^{421,436}$, Dan Arking ${ }^{437}$ Thomas G. Schulze ${ }^{438,439,375,440,441}$, Robert C. Thompson ${ }^{442,443}$ Jana Strohmaier ${ }^{375,444}$, Keith Matthews ${ }^{445,446}$, Ingrid Melle ${ }^{447,448}$ Derek Morris ${ }^{449}$, Douglas Blackwood ${ }^{450}$, Andrew McIntosh ${ }^{450}$, Sarah E. Bergen ${ }^{422}$, Martin Schalling ${ }^{451,452}$, Stéphane Jamain ${ }^{411,412,413}$ Anna Maaser ${ }^{90,89}$. Sascha B. Fischer ${ }^{94,458}$, Céline S. Reinbold ${ }^{94,458}$ Janice M. Fullerton ${ }^{454,455}$, José Guzman-Parra ${ }^{456,457}$,

Fermin Mayoral ${ }^{456,457}$, Peter R. Schofield ${ }^{454,455}$, Sven Cichon ${ }^{94,458,460}$ Thomas W. Mühleisen ${ }^{460,458}$. Franziska Degenhardt ${ }^{461}$. Johannes Schumacher ${ }^{461}$, Michael Bauer ${ }^{462}$, Philip B. Mitchell ${ }^{463,464}$ Elliot S. Gershon ${ }^{465}$, John Rice ${ }^{466}$, James B. Potash ${ }^{440}$, Peter P. Zandi ${ }^{467}$, Nick Craddock ${ }^{84}$, I. Nicol Ferrier ${ }^{418}$, Martin Alda ${ }^{468,469}$

Guy A. Rouleau ${ }^{470,471}$, Gustavo Turecki ${ }^{472}$, Roel Ophoff ${ }^{474,475}$, Carlos Pato ${ }^{476}$, Adebayo Anjorin ${ }^{473}$, Eli Stahl ${ }^{272,317}$. Markus Leber ${ }^{477}$ Piotr M. Czerski ${ }^{478}$, Cristiana Cruceanu ${ }^{479,480}$, Ian R. Jones ${ }^{481}$, Danielle Posthuma ${ }^{482,483}$, Till F.M. Andlauer ${ }^{149,484}$ Andreas J. Forstner ${ }^{90,89,95,94,486}$, Fabian Streit ${ }^{375}$, Bernhard T. Baune ${ }^{487}$, Tracy Air ${ }^{487}$, Grant Sinnamon ${ }^{489,490}$ Naomi R. Wray ${ }^{144,488}$, Donald J. Maclntyre ${ }^{491}$, David Porteous ${ }^{492}$, Georg Homuth ${ }^{493}$, Margarita Rivera ${ }^{494,276}$, Jakob Grove ${ }^{163,379,378,495}$ Christel M. Middeldorp ${ }^{496,497,128}$, Ian Hickie ${ }^{498}$. Michele Pergadia ${ }^{120}$, Divya Mehta ${ }^{499,500}$, Johannes H. Smit ${ }^{142,502,503}$, Rick Jansen ${ }^{142}$, Eco de Geus ${ }^{128,502}$, Erin Dunn ${ }^{11,501}$, Qingqin S. Li ${ }^{504}$, Matthias Nauck ${ }^{505,506}$, Robert A. Schoevers ${ }^{507}$, Aartjan TF Beekman ${ }^{142,508}$, James A. Knowles ${ }^{509}$ Alexander Viktorin $^{422}$, Paul Arnold ${ }^{510,511,424}$, Cathy L. Barr ${ }^{512,388,387}$ Gabriel Bedoya-Berrio ${ }^{513}, 0$. Joseph Bienvenu ${ }^{514}$, Helena Brentani ${ }^{515}$, Christie Burton $^{388}$, Beatriz Camarena ${ }^{516}$, Carolina Cappi ${ }^{515}$, Danielle Cath ${ }^{518,519}$, Maria Cavallini ${ }^{520}$, Daniele Cusi ${ }^{521}$, Sabrina Darrow ${ }^{522}$, Damiaan Denys ${ }^{524,525}$, Eske M. Derks ${ }^{92}$ Andrea Dietrich ${ }^{517,526}$, Thomas Fernandez ${ }^{523}$, Martijn Figee ${ }^{524,325}$ Nelson Freimer ${ }^{474}$, Gloria Gerber ${ }^{11}$, Marco Grados ${ }^{440}$,

Erica Greenberg ${ }^{119}$, Gregory L. Hanna ${ }^{443}$, Andreas Hartmann ${ }^{527,528,529}$, Matthew E. Hirschtritt ${ }^{393,395}$, Pieter J. Hoekstra ${ }^{526}$ Alden Huang ${ }^{530,390}$, Chaim Huyser ${ }^{531,532}$, Cornelia IIImann ${ }^{119}$, Michael Jenike ${ }^{13}$, Samuel Kuperman ${ }^{533}$, Bennett Leventhal ${ }^{522}$ Christine Lochner ${ }^{534}$, Gholson J. Lyon ${ }^{535}$, Fabio Macciardi536', Marcos Madruga-Garrido ${ }^{538}$, Irene A. Malaty ${ }^{537}$. Athanasios Maras ${ }^{539}$, Lauren McGrath ${ }^{540}$, Eurípedes C. Miguel $\left.\right|^{541}$. Pablo Mir ${ }^{542,543}$, Gerald Nestadt ${ }^{440}$, Humberto Nicolini ${ }^{544,545}$, Michael S. Okun ${ }^{546,547,548,}$ Andrew Pakstis ${ }^{416}$. Peristera Paschou 550 John Piacentini390 Christopher Pittenger ${ }^{549}$, Kerstin Plessen ${ }^{551.552}$, Vasily Ramensky $y^{553}$, Eliana M. Ramos ${ }^{554}$, Victor Reus ${ }^{393,395}$, Margaret A. Richter ${ }^{555,556}$ Mark A. Riddle ${ }^{514}$, Mary M. Robertson ${ }^{557}$, Veit Roessner ${ }^{558}$ Maria Rosário ${ }^{559,560}$, Jack F. Samuels ${ }^{440}$, Paul Sandor ${ }^{556,561,562}$ Dan J. Stein ${ }^{563,534}$, Fotis Tsetsos ${ }^{564}$, Filip Van Nieuwerburgh ${ }^{565}$ Sarah Weatherall11, Jens R. Wendland ${ }^{566}$, Tomasz Wolanczyk ${ }^{56}$ Yulia Worbe ${ }^{568,569,570}$, Gwyneth Zai ${ }^{556}$, Fernando S. Goes ${ }^{440}$, Nicole McLaughlin ${ }^{571,572}$, Paul S. Nestadt ${ }^{440}$, Hans-Jorgen Grabe ${ }^{573}$, Christel Depienne ${ }^{574,575,576}$, Anuar Konkashbaev ${ }^{577}$ Nuria Lanzagorta ${ }^{545}$, Ana Valencia-Duarte ${ }^{578,579}$, Elvira Bramon ${ }^{98}$, Nancy Buccola ${ }^{580}$. Wiepke Cahn ${ }^{581}$. Murray Cairns ${ }^{582,583,584}$, Siow A. Chong ${ }^{585}$, David Cohen ${ }^{586,587}$, Benedicto Crespo-Facorro ${ }^{588,348}$ James Crowley ${ }^{288}$, Michael Davidson ${ }^{589,590}$, Lynn DeLisi ${ }^{591,13}$ Timothy Dinan ${ }^{592,593}$, Gary Donohoe ${ }^{594}$, Elodie Drapeau ${ }^{272,325,326}$, Jubao Duan ${ }^{595,596}$, Lieuwe Haan ${ }^{524,597}$, David Hougaard ${ }^{598}$, Sena Karachanak-Yankova ${ }^{599}$, Andrey Khrunin ${ }^{600}$, Janis Klovins ${ }^{601}$, Vaidutis Kučinskas $^{602}$, Jimmy Lee Chee Keong ${ }^{603}$ Svetlana Limborska ${ }^{604}$, Carmel Loughland ${ }^{605,250}$ Jouko Lönnqvist ${ }^{154,606}$, Brion Maher ${ }^{467}$, Manuel Mattheisen ${ }^{607,608,609}$ Colm McDonald ${ }^{610,611}$, Kieran C. Murphy ${ }^{612}$, Igor Nenadic ${ }^{613,614}$, Jim van Os ${ }^{581,615,616}$, Christos Pantelis ${ }^{617,618,219}$, Michele Pato 476, Tracey Petryshen 2,11 . Digby Quested ${ }^{619,620}$. Panos Roussos ${ }^{272}$ Alan R. Sanders ${ }^{621,596}$, Ulrich Schall ${ }^{250}$, Sibylle G. Schwab ${ }^{622}$ Kang Sim ${ }^{623,624,585}$, Hon-Cheong So ${ }^{625,626,627}$, Elisabeth Stögmann ${ }^{237}$, Mythily Subramaniam ${ }^{585,624}$, Draga Toncheva ${ }^{599}$, John Waddington ${ }^{202}$, James Walters ${ }^{84,85}$, Mark Weiser ${ }^{590,628}$, Wei Cheng ${ }^{271}$. Robert Cloninger ${ }^{629}$, David Curtis ${ }^{630,631}$ Pablo V. Gejman ${ }^{596,621}$. Frans Henskens ${ }^{632,633,251}$ Morten Mattingsdal ${ }^{447,634}$, Sang-Yun $0{ }^{635,636}$, Rodney Scott ${ }^{250,251,637}$, Bradley Webb ${ }^{638}$, Gerome Breen ${ }^{639,640}$, Claire Churchhouse ${ }^{1,2,3}$, Cynthia M. Bulik ${ }^{641,422}$, Mark Daly ${ }^{1,2,3}$, Martin Dichgans ${ }^{14,150}$ Stephen V. Faraone ${ }^{642}$, Rita Guerreiro ${ }^{6,7}$. Peter Holmans ${ }^{9}$, Kenneth S. Kendler ${ }^{643}$, Bobby Koeleman ${ }^{644}$, Carol A. Mathews ${ }^{645.548}$, Alkes Price ${ }^{3,5}$, Jeremiah Scharf ${ }^{2,3,11,646,12,13}$, Pamela Sklar272, Julie Williams ${ }^{9,10}$, Nicholas W. Wood ${ }^{7,630}$, Chris Cotsapas ${ }^{3,647}$ Aarno Palotie $1,2,3,11,12,13,123$, Jordan W. Smoller, 2,11 . Patrick Sullivan ${ }^{641,648}$, Jonathan Rosand ${ }^{3,11,12,13}$, 'Aiden Corvin ${ }^{2,649 *}+$ Benjamin M. Neale $\mathrm{I}^{1,2,3 *} \dagger$

${ }^{1}$ Analytic Translational Genetics Unit, Massachusetts General Hospital and Harvard Medical School, Boston, Massachusetts, USA. ${ }^{2}$ Stanley Center for Psychiatric Research, Broad Institute of MIT and 
Harvard, Cambridge, Massachusetts, USA. ${ }^{3}$ Program in Medical and Population Genetics, Broad Institute of MIT and Harvard, Cambridge Massachusetts, USA. ${ }^{4}$ Department of Mathematics, Massachusetts Institute of Technology, Cambridge, Massachusetts, USA. ${ }^{5}$ Department of Epidemiology, Harvard T.H. Chan School of Public Health, Boston, Massachusetts, USA. ${ }^{6}$ UK Dementia Research Institute, University College London, London, UK. 'Department of Molecular Neuroscience, Institute of Neurology, University College London, London, UK. ${ }^{8}$ Department of Psychiatry and Behavioral Science, Stanford University, Stanford, California, USA. ${ }^{9}$ Cardiff University, Medical Research Council Center for Neuropsychiatric Genetics \& Genomics, Institute of Psychology, Medicine \& Clinical Neuroscience, Cardiff, UK. ${ }^{10}$ Dementia Research Institute, Cardiff University, Cardiff, UK. ${ }^{11}$ Center for Genomic Medicine, Massachusetts General Hospital, Boston, MA, USA. ${ }^{12}$ Department of Neurology, Massachusetts General Hospital, Boston, MA, USA. ${ }^{13}$ Harvard Medical School, Boston, MA, USA. ${ }^{14}$ Institute for Stroke and Dementia Research (ISD), University Hospital, LMU Munich, Munich, Germany. ${ }^{15}$ Department of Neurology, Brigham \& Women's Hospital, Harvard Medical School, Boston, MA. USA. ${ }^{16}$ Charite Universitatsmedizin Berlin, Berlin, Germany. ${ }^{17}$ Department of Computer Science, New Jersey Institute of Technology, New Jersey, USA. ${ }^{18}$ INSERM U1167 LabEx DISTALZ, Lille, France. ${ }^{19}$ Institut Pasteur de Lille, U1167, Lille, France. ${ }^{20}$ Université de Lille, U1167, RID-AGE, Risk Factors and Molecular Determinants of Aging-Related Diseases, Lille, France. ${ }^{21}$ Centre Hosp. Univ Lille, Lille, France. ${ }^{22}$ Laboratory for Statistical Analysis, RIKEN Center for Integrative Medical Sciences, Yokohama, Japan. ${ }^{23}$ Center for Genomic Medicine, Kyoto University Graduate School of Medicine, Kyoto, Japan. ${ }^{24}$ INSERM U1061 - Neuropsychiatry: Epidemiological and Clinical Research, Montpellier, France. ${ }^{25}$ University of Montpellier, Montpellier, France. ${ }^{26}$ Memory Research and Resources Center, Department of Neurology, Montpellier University Hospital Gui de Chauliac, Montpellier, France. ${ }^{27}$ INSERM, UMR 1219, Bordeaux, France. ${ }^{28}$ University of Bordeaux, Bordeaux, France. ${ }^{29}$ Rouen University Hospital, Rouen, France. ${ }^{30}$ Inserm U1245, Rouen, France. ${ }^{31}$ Centre National de Recherche en Génomique Humaine (CNRGH), Institut de biologie François Jacob, CEA, Evry, France. ${ }^{32}$ Department of Gerontology, Hôpital Broca, AH-HP, Paris, France. ${ }^{33}$ Hôpital Paul Brousse Université Paris Sud XI, Le Kremlin-Bicêtre, Paris, France. ${ }^{34}$ Gertrude H. Sergievsky Center and Dept of Neurology, Columbia University, New York, NY, USA. ${ }^{35}$ Columbia University, New York, NY, USA. ${ }^{36}$ Department of Neuroscience, Icahn School of Medicine at Mount Sinai, New York, NY, USA. ${ }^{37}$ Translational Genomics Research Institute, Neurogenomics Division, Phoenix, AZ, USA. ${ }^{38}$ University of Pittsburgh, Pittsburgh, PA, USA. ${ }^{39}$ Kaiser Permanente Washington Health Research Institute, Seattle, WA, USA. ${ }^{40}$ Department of Medicine, University of Washington, WA, USA. ${ }^{41}$ Tanz Centre for Research in Neurodegenerative Diseases, University of Toronto Toronto, Canada. ${ }^{42}$ Cambridge Institute for Medical Research, University of Cambridge, Cambridge, UK. ${ }^{43}$ Center for Applied Genomics of The Children's Hospital of Philadelphia, Philadelphia, PA USA. ${ }^{44}$ Division of Human Genetics, Children's Hospital of Philadelphia, Philadelphia, PA, USA. ${ }^{45}$ Department of Pediatrics, The Perelman School of Medicine, University of Pennsylvania, Philadelphia, PA, USA. ${ }^{46}$ National Alzheimer Coordinating Center (NACC), Department of Epidemiology, University of Washington, Seattle, WA, USA. ${ }^{47}$ Department of Medicine, Boston University School of Medicine Boston, MA, USA. ${ }^{48}$ Rush Alzheimers Disease Center, Chicago, IL, USA. ${ }^{49}$ Department of Neurological Sciences, Rush Medical College, Chicago, IL, USA. ${ }^{50}$ Department of Behavioral Sciences, Rush Medical College, Chicago, IL, USA. ${ }^{51}$ Banner Sun Health Research Institute, Sun City, AZ, USA. ${ }^{52}$ Sanders-Brown Center on Aging, University of Kentucky, Lexington, KY, USA. ${ }^{53}$ Department of Pathology, Duke University School of Medicine, Durham, NC, USA. ${ }^{54}$ College of Medicine, University of Kentucky, Lexington, KY, USA. ${ }^{55}$ Department of Biology, Brigham Young University, Provo, UT, USA. ${ }^{56}$ Layton Aging \& Alzheimer's Disease Center, Oregon Health \& Science University, Portland, OR, USA. ${ }^{57}$ Lou Ruvo Center for Brain Health, Neurological Institute, Cleveland Clinic, Cleveland, OH, USA. ${ }^{58}$ Department of Neurology, School of Medicine, Emory University, Atlanta, GA, USA. ${ }^{59}$ Department of Pathology, University of Michigan Medical School, Ann Arbor, MI, USA. ${ }^{60}$ University of New Mexico Health Sciences Center, Albuquerque, NM, USA. ${ }^{61}$ Institute for Memory Impairments and Neurological Disorders, University of California, Irvine, CA, USA. ${ }^{62}$ Department of Neurology, Oregon Health and Science University, Portland, OR, USA. ${ }^{63}$ Department of Neurology and Parkinson's Disease Research Education and Clinical Care Center (PADRECC), Portland Veterans Affairs Medical Center, Portland, OR, USA. ${ }^{64}$ Indiana Alzheimer Disease Center, Indiana University School of Medicine, Indianapolis, IN, USA. ${ }^{65}$ Keck School of Medicine of the University of Southern California, Los Angeles, CA, USA. ${ }^{66}$ Byrd Alzheimer's Institute, University of South Florida, Tampa, FL, USA. ${ }^{67}$ Department of Pathology, University of Utah, Salt Lake City, UT, USA.
${ }^{68}$ Department of Pathology, University of Washington, Seattle, WA, USA. ${ }^{69}$ Boston University School of Medicine, Boston, MA, USA ${ }^{70}$ Department of Pathology and Laboratory Medicine, Perelman School of Medicine, University of Pennsylvania, Philadelphia, PA, USA. ${ }^{71}$ School of Biotechnology, Dublin City University, Glasnevin, Dublin, Ireland. ${ }^{72}$ Functional Genomics Center Zurich, ETH/UZH-Zurich, Zurich, Switzerland. ${ }^{73}$ Department of Medical Genetics, Cambridge Institute for Medical Research, Cambridge, UK. ${ }^{74}$ UK Dementia Research Institute, Cambridge, UK. ${ }^{75}$ School of Medicine, Cardiff University, Cardiff, UK. ${ }^{76} 1$ st and 3rd Departments of Neurology, Aristotle University of Thessaloniki, Thessaloniki, Greece. ${ }^{77}$ Greek Association of Alzheimer's Disease and Related Disorders,

Thessaloniki, Greece. ${ }^{78}$ Maurice Wohl Clinical Neuroscience Institute, Institute of Psychiatry, Psychology and Neuroscience, King's College London, London, UK. ${ }^{79}$ Dementia Research Centre, UCL Institute of Neurology, London, UK. ${ }^{80}$ Sorbonne University, GRC n 21, Alzheimer Precision Medicine (APM), AP-HP, Pitié-Salpêtrière Hospital, Paris, France. ${ }^{81}$ Institute of Memory and Alzheimer's Disease (IM2A), Department of Neurology, Pitié-Salpêtrière Hospital, AP-HP, Paris, France. ${ }^{82}$ Brain \& Spine Institute (ICM), INSERM U 1127, CNRS UMR 7225, Paris, France. ${ }^{83}$ AXA Research Fund \& Sorbonne University Chair, Paris, France. ${ }^{84} \mathrm{MRC}$ Centre for Neuropsychiatric Genetics and Genomics, Cardiff University, Cardiff, UK. ${ }^{85}$ Institute of Psychological Medicine and Clinical Neurosciences, School of Medicine, Cardiff University, Cardiff, UK. ${ }^{86}$ Institute of Prion Diseases and MRC Prion Unit, University College London, London, UK. ${ }^{87}$ Centre for Public Health, Queens University Belfast, Belfast, UK. ${ }^{88}$ Human Genetics, School of Life Sciences, University of Nottingham, Nottingham UK. ${ }^{89}$ Department of Genomics, Life \& Brain Center, University of Bonn, Bonn, Germany. ${ }^{90}$ Institute of Human Genetics, School of Medicine. University of Bonn \& University Hospital Bonn, Bonn, Germany. ${ }^{91}$ Department of Neurodegeneration, UCL Institute of Neurology, London, UK. ${ }^{92}$ QIMR Berghofer Medical Research Institute, Brisbane, Australia. ${ }^{93}$ Institute of Psychiatry Psychology and Neuroscience Kings College London, UK. ${ }^{94}$ Institute of Medical Genetics and Pathology, University Hospital Basel, Basel, Switzerland. ${ }^{95}$ Human Genomics Research Group, Department of Biomedicine, University of Basel, Basel, Switzerland. ${ }^{96}$ Department of Psychiatry and Psychotherapy, Friedrich-Alexander-Universität Erlangen-Nürnberg University Hospital, Erlangen, Germany. ${ }^{97}$ Department of Psychiatry and Global Brain Health Institute, Trinity College, Dublin, Ireland. ${ }^{98}$ Division of Psychiatry, Molecular Psychiatry Laboratory, University College London, London, UK. ${ }^{99}$ Maurice Wohl Clinical Neuroscience Institute, Department of Basic and Clinical Neuroscience, King's College London, London, UK. ${ }^{100}$ King's College Hospital, London, UK. ${ }^{101}$ Cardiovascular Health Research Unit, Department of Medicine, University of Washington, Seattle, WA, USA. ${ }^{102}$ Fundació ACE, Institut Català de Neurociències Aplicades, Barcelona, Spain and Universitat Internacional de Catalunya, Barcelona, Spain. ${ }^{103}$ Facultat de Medicina i Ciències de la Salut, Universitat Internacional de Catalunya (UIC). Barcelona, Spain. ${ }^{104}$ Glenn Biggs Institute for Alzheimer's and Neurodegenerative Diseases, University of Texas Health Sciences Center, San Antonio, Texas, USA. ${ }^{105}$ Neurology and Neurogenetics Core, Framingham Heart Study, Framingham, MA, USA. ${ }^{106}$ School of Medicine, Boston University, Boston, MA, USA. ${ }^{107}$ School of Public Health, Boston University, Boston, MA, USA. ${ }^{108}$ Framingham Heart Study, Framingham, MA, USA. ${ }^{109}$ Department of Biostatistics, University of Washington, Seattle, WA, USA. ${ }^{110}$ Department of Epidemiology, Erasmus Medical Centre, Rotterdam, the Netherlands. ${ }^{111}$ Center for Translational \& Computational Neuroimmunology, Columbia University Medical Center, New York, NY, USA. ${ }^{112}$ Neurogenetics Program, Departments of Neurology and Human Genetics, David Geffen School of Medicine, University of California, Los Angeles, Los Angeles, CA, USA. ${ }^{113}$ Center For Autism Research and Treatment, Semel Institute, David Geffen School of Medicine, University of California, Los Angeles, Los Angeles, CA, USA. ${ }^{114}$ Institute for Precision Health, University of California, Los Angeles, Los Angeles, CA, USA. ${ }^{115}$ Department of Psychiatry, Saarland University Hospital, Homburg, Germany. ${ }^{116}$ Institute of Social Medicine, Occupational Health and Public Health (ISAP), University of Leipzig, Leipzig. Germany. ${ }^{117}$ Institute for Translational Genomics and Population Sciences, Departments of Pediatrics and Medicine, LABioMed at Harbor-UCLA Medical Center, Torrance, CA, USA. ${ }^{118}$ Department of Neurology II, Kepler University Clinic, Johannes Kepler University, Linz, Austria. ${ }^{119}$ Massachusetts General Hospital, Boston, MA, USA. ${ }^{120}$ Washington University School of Medicine, St. Louis, MO, USA ${ }^{121}$ Communication and Research Unit for Musculoskeletal Disorders (FORMI), Oslo University Hospital, Oslo, Norway. ${ }^{122}$ Department of Neurology, Oslo University Hospital, Oslo, Norway. ${ }^{123}$ Institute for Molecular Medicine Finland (FIMM), HiLIFE, University of Helsinki, Helsinki, Finland. ${ }^{124}$ Estonian Genome Center, Institute of Genomics, University of Tartu, Tartu, Estonia. ${ }^{125}$ Illumina Inc., San Diego, CA, USA. ${ }^{126} 23$ andMe Inc., Mountain View, CA, USA. ${ }^{127}$ Institute of Public
Health, Charité - Universitätsmedizin Berlin, Berlin, Germany. ${ }^{128}$ Department of Biological Psychology, Vrije Universiteit Amsterdam Amsterdam, The Netherlands. ${ }^{129}$ Department of Neurology, Leiden University Medical Center, Leiden, The Netherlands. ${ }^{130}$ Institute for Stroke and Dementia Research, Klinikum der Universitaet Muenchen, Munich, Germany. ${ }^{131}$ Department of Neurology and Epileptology, Hertie Institute for Clinical Brain Research, University of Tuebingen Tuebingen, Germany. ${ }^{132}$ Department of Neuroscience, Karolinska Institutet, Stockholm, Sweden. ${ }^{133}$ Institute of Human Genetics, University of Ulm, Ulm, Germany. ${ }^{134}$ Department of Radiology and Nuclear Medicine, Erasmus Medical Centre, Rotterdam, the Netherlands. ${ }^{135}$ Department of Clinical Chemistry, Fimlab Laboratories and Finnish Cardiovascular Research Center-Tampere, Faculty of Medicine and Life Sciences, University of Tampere, Tampere, Finland. ${ }^{136}$ Department of Ophthalmology, University of Helsinki and Helsinki University Hospital, Helsinki, Finland. ${ }^{137}$ Department of Public Health, University of Helsinki, Helsinki, Finland. ${ }^{138}$ Brigham and Women's Hospital, Boston, MA. ${ }^{139}$ Department of Neurology, University Hospital Essen, Germany. ${ }^{140}$ Landspitali National University Hospital, Reykjavik, Iceland. ${ }^{141}$ Avera Institute for Human Genetics, Sioux Falls, SD, USA. ${ }^{142}$ Department of Psychiatry, VU University Medical Center Amsterdam, The Netherlands. ${ }^{143}$ Department of Neurology, Helsinki University Central Hospital, Helsinki, Finland. ${ }^{144}$ Institute for Molecular Bioscience, University of Queensland, Brisbane, Australia.

${ }^{145}$ Department of Medicine, Harvard Medical School, Boston, MA, USA. ${ }^{146}$ Boston VA Research Institute, Boston, MA, USA. ${ }^{147}$ Brigham Women's Hospital Division of Aging, Harvard Medical School, Boston, MA, USA. ${ }^{148}$ Department of Physiology, Anatomy and Genetics, University of Oxford, Oxford, UK. ${ }^{149}$ Max Planck Institute of Psychiatry, Munich, Germany. ${ }^{150}$ Munich Cluster for Systems Neurology (SyNergy), Munich, Germany. ${ }^{151}$ Institute of Translational Medicine, University of Liverpool, Liverpool, UK. ${ }^{152}$ Institute of Clinical Molecular Biology, Kiel University and University Hospital Schleswig-Holstein, Kiel, Germany. ${ }^{153} \mathrm{Clinic}$ of Internal Medicine I, University Hospital Schleswig-Holstein, Kiel, Germany. ${ }^{154}$ National Institute for Health and Welfare, Helsinki, Finland. ${ }^{155}$ Department of Environmental Health, Harvard T.H. Chan School of Public Health, Boston, MA, USA ${ }^{156}$ Kiel Pain and Headache Center, Kiel, Germany. ${ }^{157}$ Pediatric Neurology Research Group, Vall d'Hebron Research Institute, Autonomous University of Barcelona, Barcelona, Spain. ${ }^{158}$ Headache Unit, Neurology Department, Hospital Vall d'Hebron, Barcelona, Spain. ${ }^{159}$ Headache Research Group, VHIR, Autonomous University of Barcelona, Barcelona, Spain. ${ }^{160}$ Danish Headache Center, Rigshospitalet Glostrup and University of Copenhagen, Copenhagen, Denmark. ${ }^{161}$ Institute of Biological Psychiatry, Roskilde, Denmark. ${ }^{162}$ Department of Clinical Sciences, University of Copenhagen, Copenhagen, Denmark. ${ }^{163}$ Lundbeck Foundation Initiative for Integrative Psychiatric Research, iPSYCH, Aarhus, Denmark. ${ }^{164}$ Institute of Human Genetics, University Medical Center Hamburg-Eppendorf, Hamburg, Germany. ${ }^{165}$ Karolinska Institutet, Stockholm, Sweden. ${ }^{166}$ Department of Human Genetics, Leiden University Medical Center, Leiden, The Netherlands. ${ }^{167}$ Division of Clinical Neuroscience, Oslo University Hospital and University of Oslo, Oslo, Norway. ${ }^{168}$ Netherlands Twin Register, Vrije Universiteit, Amsterdam, the Netherlands. ${ }^{169}$ Division of Preventive Medicine, Brigham and Women's Hospital, Boston, MA, USA. ${ }^{170}$ Statistical and Genomic Epidemiology Laboratory, Institute of Health and Biomedical Innovation, Queensland University of Technology, Brisbane, Queensland, Australia. ${ }^{171}$ Department of Clinical and Experimental Epilepsy, UCL Institute of Neurology, London, UK. ${ }^{172}$ Centre for Genomic Sciences, University of Hong Kong, Hong Kong. ${ }^{173}$ Epilepsy Research Centre, University of Melbourne, Heidelberg, Australia. ${ }^{174}$ Quantinuum Research LLC, San Diego, CA, USA. ${ }^{175}$ Cooper Medical School of Rowan University, Camden, NJ, USA. ${ }^{176}$ Thomas Jefferson University Hospital, Philadelphia, PA, USA. ${ }^{177}$ Children's Hospital of Philadelphia, Philadelphia, PA, USA. ${ }^{178}$ Epilepsy Society, Chalfont-St-Peter, Bucks, UK. ${ }^{179}$ Centre de Recherche du Centre Hospitalier de l'Universite de Montreal and Department of Neurosciences, Université de Montréal, Montréal, Canada. ${ }^{180}$ Neurogenetics Group, VIB-CMN, Antwerp, Belgium. ${ }^{181}$ University of Antwerp, Antwerp, Belgium. ${ }^{182}$ Department of Neurology, Antwerp University Hospital, Antwerp, Belgium. ${ }^{183}$ Department of Neurology, Hôpital Erasme, Université Libre de Bruxelles, Brussels, Belgium. ${ }^{184}$ Division of Neurology, Children's Hospital of Philadelphia, Philadelphia, PA, USA. ${ }^{185}$ Perelman School of Medicine at the University of Pennsylvania, Philadelphia, PA, USA. ${ }^{186}$ Department of Biomedical Sciences, Cooper Medical School of Rowan University, Camden, NJ, USA. ${ }^{187}$ Department of Psychiatry, Center for Neurobiology and Behavior, Perelman School of Medicine at the University of Pennsylvania, Philadelphia, PA, USA. ${ }^{188} \mathrm{NYU}$ School of Medicine, New York, NY, USA. ${ }^{189}$ Amplexa Genetics A/S, Odense, Denmark. ${ }^{190}$ Institute of Mental Health, University of Nottingham, Nottingham, UK. ${ }^{191}$ Human Genetics, School of Life Sciences, 
University of Nottingham, Nottingham UK. ${ }^{192}$ Epilepsy Center/ Neurocenter, Kuopio University Hospital, Kuopio, Finland. ${ }^{193}$ Institute of Clinical Medicine, School of Medicine, Faculty of Health Sciences, University of Eastern Finland, Kuopio, Finland. ${ }^{194}$ Department of Epileptology, University Bonn Medical Center, Bonn, Germany. ${ }^{195}$ Institute of Experimental Epileptology and Cognition Research, University Bonn Medical Center, Bonn, Germany. ${ }^{196}$ Department of Clinical and Experimental Epilepsy, NIHR University College London Hospitals Biomedical Research Centre, UCL Institute of Neurology, London. ${ }^{197}$ Department of Genetics, University Medical Center Utrecht, the Netherlands. ${ }^{198}$ Epilepsy Foundation in the Netherlands (SEIN), Heemstede, the Netherlands. ${ }^{199}$ Departments of Pediatrics and Neurology, Ohio State University, Columbus, $\mathrm{OH}$, USA

${ }^{200}$ Nationwide Children's Hospital, Columbus, OH, USA.

${ }^{201}$ Department of Neurology, University of California, San Francisco, CA, USA. ${ }^{202}$ Department of Molecular and Cellular Therapeutics, Royal College of Surgeons in Ireland, Dublin, Ireland. ${ }^{203}$ Center for Molecular Medicine, University Medical Center Utrecht, Utrecht, the Netherlands. ${ }^{204}$ Danish Epilepsy Centre, Filadelfia, Dianalund, Denmark. ${ }^{205}$ Institute for Regional Health Services, University of Southern Denmark, Odense, Denmark. ${ }^{206}$ Trinity College Dublin, Dublin, Ireland. ${ }^{207}$ United Christian Hospital, Hong Kong. ${ }^{208}$ Hong Kong Sanatorium and Hospital, Hong Kong. ${ }^{209}$ Epilepsy Research Centre, University of Melbourne, Austin Health, Heidelberg, Australia. ${ }^{210}$ Department of Neurology, University of Cincinnati, Cincinnati, OH, USA. ${ }^{211}$ UC Gardner Neuroscience Institute, Cincinnati, OH, USA. ${ }^{212}$ Department of Neurology, Duke University School of Medicine, Durham, NC, USA. ${ }^{213}$ Cologne Center for Genomics (CCG), University of Cologne, Cologne, Germany. ${ }^{214}$ Department of Neurology, Beth Israel Deaconess Medical Center, Boston, MA, USA. ${ }^{215}$ Department of Neurology, Inselspital, Bern University Hospital, University of Bern, Switzerland. ${ }^{216}$ Department of Neurology. University of Munich Hospital, Grosshadern, University of Munich, Germany. ${ }^{217}$ Department of Medicine, The University of Melbourne, Austin Health, Melbourne Victoria, Australia. ${ }^{218}$ Department of Paediatrics, Royal Children's Hospital, The University of Melbourne, Melbourne, Victoria, Australia. ${ }^{219}$ Florey Institute of Neuroscience and Mental Health, Melbourne, Victoria, Australia. ${ }^{220}$ Institute of Neuropathology, Bonn University Medical School, Bonn, Germany. ${ }^{221}$ UCL Institute of Neurology, London, UK. ${ }^{222}$ Chalfont Centre for Epilepsy, Bucks, UK. ${ }^{223}$ University Hospital of Wales, Cardiff, UK. ${ }^{224}$ Department of Neurology, Thomas Jefferson University, Philadelphia, PA, USA. ${ }^{225}$ Pediatric Neurology and Muscular Diseases Unit-Department of Neurosciences, Rehabilitation, Ophthalmology, Genetics, Maternal and Child Health University of Genoa, "G. Gaslini" Institute, Genova, Italy. ${ }^{226}$ Department of Epileptology, University Hospital Bonn, Bonn, Germany. ${ }^{227}$ Section of Epileptology, Department of Neurology, University Hospital RWTH Aachen, Aachen, Germany. ${ }^{228}$ Institute of Applied Health Research, University of Birmingham, UK. ${ }^{229}$ Department of Neurology, Admiraal De Ruyter Hospital, Goes, The Netherlands. ${ }^{230}$ Laboratory of Neurogenetics, G. Gaslini Institute, Genova, Italy. ${ }^{231}$ Institute for Genomic Medicine, Columbia University Medical Center, New York, NY, USA. ${ }^{232}$ University of Liverpool, Liverpool, UK. ${ }^{233}$ Walton Centre NHS Foundation Trust, Liverpool, UK. ${ }^{234}$ Department of Neurology and Epileptology, University Hospital Tuebingen, Tuebingen, Germany. ${ }^{235}$ Department of Neurology, University of Ulm, Ulm, Germany. ${ }^{236} \mathrm{CWZ}$ Hospital, Nijmegen, Netherlands. ${ }^{237}$ Department of Neurology, Medical University of Vienna, Austria. ${ }^{238}$ Hertie-Institute for Clinical Brain Research, University of Tübingen, Tübingen, Germany. ${ }^{239}$ German Center for Neurodegenerative Diseases (DZNE), Tübingen, Germany. ${ }^{240}$ Laboratory of Neurogenetics, National Institute on Aging, National Institutes of Health, Bethesda, MD, USA. ${ }^{241}$ INSERM U1220, IRSD, Toulouse, France. ${ }^{242}$ Université Paul Sabatier, Toulouse, France. ${ }^{243}$ Centre for Genetic Epidemiology, Institute for Clinical Epidemiology and Applied Biometery, University of Tubingen, Germany. ${ }^{244}$ Department of Molecular Neuroscience, Institute of Neurology, University College London, London, UK. ${ }^{245}$ Division of Life Science, Hong Kong University of Science and Technology, Hong Kong Special Administrative Region, China. ${ }^{246}$ Department of Genetics, Center for Molecular Medicine, University Medical Center Utrecht, Utrecht, The Netherlands. ${ }^{247}$ Big Data Institute, Li Ka Shing Centre for Health Information and Discovery, University of Oxford, Oxford, UK. ${ }^{248}$ University of Lincoln, Lincoln, UK. ${ }^{249}$ Faculty of Health and Medicine, University of Newcastle, Callaghan, Australia. ${ }^{250}$ University of Newcastle, Callaghan, Australia. ${ }^{251}$ Hunter Medical Research Institute, Newcastle, Australia. ${ }^{252}$ Center for Genomic Medicine, Massachusetts General Hospital, Boston, MA, USA. ${ }^{253}$ Division of Neurocritical Care and Emergency Neurology, Massachusetts General Hospital, Boston, MA, USA. ${ }^{254}$ Department of Cerebrovascular Diseases, Fondazione IRCCS Istituto Neurologico Carlo Besta, Milano, Italy. ${ }^{255} \mathrm{PhD}$ Program in Neuroscience, University Milano-Bicocca, Monza, Italy. ${ }^{256}$ Austin Health, Heidelberg, Australia. ${ }^{257}$ University of Virginia Center for Public Health Genomics, University of Virginia,
Charlottesville, VA, USA. ${ }^{258}$ Dept of Medicine, University of Marylan School of Medicine, Baltimore, MD, USA. ${ }^{259}$ Geriatrics Research and Education Clinical Center, Baltimore Veterans Administration Medical Center, Baltimore, MD, USA. ${ }^{260}$ Centre for Prevention of Stroke and Dementia, Nuffield Department of Clinical Neurosciences, University of Oxford, Oxford, UK. ${ }^{261}$ Institute of Cardiovascular Research, Royal Holloway University of London, London, UK. ${ }^{262}$ Ashford \& St Peters NHS Foundation Trust, Surrey, UK. ${ }^{263}$ University of Edinburgh, Edinburgh, UK. ${ }^{264}$ Instituto Nacional de Saúde Doutor Ricardo Jorge, Lisboa, Portugal. ${ }^{265}$ Biosystems and Integrative Sciences Institute - BiolSI, University of Lisboa, Lisboa, Portugal. ${ }^{266}$ Department of Clinical Neurosciences, University of Cambridge, Cambridge, UK. ${ }^{267}$ Department of Neurology, Jagiellonian University Medical College, Kraków, Poland. ${ }^{268}$ The Warren Alpert Medical School of Brown University, Providence, RI, USA.

${ }^{269}$ Department of Neurology, College of Medicine-Jacksonville, University of Florida, Jacksonville, FL, USA. ${ }^{270}$ University of Split School of Medicine, Split, Croatia. ${ }^{271}$ University of North Carolina at Chapel Hill, Chapel Hill, NC, USA. ${ }^{272}$ Icahn School of Medicine at Mount Sinai, New York, NY, USA. ${ }^{273}$ Wellcome Centre for Human Genetics, Nuffield Department of Medicine, University of Oxford, Oxford, UK. ${ }^{274}$ Oxford Centre for Diabetes, Endocrinology and Metabolism, Nuffield Department of Medicine, University of Oxford, Oxford, UK. ${ }^{275}$ Department of Human Genetics, Wellcome Sanger Institute, Hinxton. Cambridgeshire, UK. ${ }^{276}$ MRC Social, Genetic and Developmental Psychiatry Centre, King's College London, London, UK. ${ }^{277}$ Genes and Disease Programme, Centre for Genomic Regulation (CRG), Barcelona, Spain. ${ }^{278}$ Department of Adult Psychiatry, Poznan University of Medical Sciences, Poland. ${ }^{279}$ Clinicum, Department of Public Health, University of Helsinki, Finland. ${ }^{280}$ Department of Adolescent Psychiatry, Helsinki University Central Hospital, Helsinki, Finland. ${ }^{281}$ Harvard Medical School/McLean Hospital, Belmont, MA, USA. ${ }^{282}$ Norwegian Institute of Public Health, Oslo, Norway. ${ }^{283}$ University of Oslo, Oslo, Norway. ${ }^{284}$ Department of Medicine Surgery and Dentistry "Scuola Medica Salernitana", University of Salerno,Italy. ${ }^{285}$ Eating Disorders Unit, Department of Child and Adolescent Psychiatry, Medical University of Vienna, Vienna, Austria. ${ }^{286}$ Center for Integrative Genomics, University of Lausanne, Lausanne Switzerland. ${ }^{287}$ Kartini Clinic, Portland, OR, USA. ${ }^{288}$ Department of Psychiatry, University of North Carolina at Chapel Hill, Chapel Hill, NC USA. ${ }^{289}$ MRC Integrative Epidemiology Unit and Bristol Medica School, University of Bristol, Bristol, UK. ${ }^{290}$ Zorg op Orde BV, Leidschendam, The Netherlands. ${ }^{291}$ Division of Psychological \& Social Medicine and Developmental Neurosciences, Faculty of Medicine, Technischen Universität Dresden, Dresden, Germany. ${ }^{292}$ Department of Child \& Adolescent Psychiatry \& Psychosomatic Medicine of University Clinics, RWTH Aachen, Aachen, Germany. ${ }^{293}$ Altrecht Eating Disorders Rintveld, Altrecht Mental Health Institute, Zeist, The Netherlands. ${ }^{294}$ Faculty of Social Sciences, University of Utrecht, Utrecht, the Netherlands. ${ }^{295}$ Medical Genetics Unit, Department SDB, University of Padova, Padova, Italy. ${ }^{296}$ UOC Genetica ed Epidemiologica Clinica Az. Ospedaliera, Padova, Italy. ${ }^{297}$ Department of Human Genetics, CHU Sart-Tilman, University of Liège, Liège, Belgium. ${ }^{298}$ Department of Rheumatology, CHU Sart-Tilman, University of Liège, Liège, Belgium. ${ }^{299}$ Department of Cancer Epidemiology and Prevention, Cancer Center and M. SklodowskaCurie Institute of Oncology, Warsaw, Poland. ${ }^{300}$ Department of Psychiatry, University of Medical Sciences, Poznan, Poland. ${ }^{301}$ Department of Psychiatry, University of Perugia, Perugia, Italy. ${ }^{302}$ Department of Mental and Physical Health and Preventive Medicine, University of Campania "luigi Vanvitelli", Naples, Italy. ${ }^{303}$ Eating Disorders Unit, 1st Psychiatric Department, National and Kapodistrian University of Athens, Athens, Greece. ${ }^{304}$ Aglaia Kyriakou Childrens Hospital, Athens, Greece. ${ }^{305}$ Eating Disorders Unit, Department of Psychiatry, First Faculty of Medicine, Charles University, Prague, Czech Republic. ${ }^{306}$ General University Hospital, Prague, Czech Republic. ${ }^{307}$ Medical University of Vienna, Austria. ${ }^{308}$ School of Psychology, Flinders University, Adelaide, Australia. ${ }^{309}$ Division of Medical Genetics, University Hospital Basel, Basel, Switzerland. ${ }^{310}$ Genomics Research Group, Department of Biomedicine, University of Basel, Basel, Switzerland. ${ }^{311}$ Vall d'Hebron Research Institute, Barcelona, Spain. ${ }^{312}$ Institut de Recerca Sant Joan de Déu, Barcelona, Spain. ${ }^{313}$ Institut de Biomedicina de la Universitat de Barcelona (IBUB), Barcelona, Spain. ${ }^{314}$ Department of Genetics, Microbiology \& Statistics, Faculty of Biology, University of Barcelona, Barcelona, Spain. ${ }^{315}$ Centre for Genomic Regulation (CRG), Barcelona, Spain. ${ }^{316}$ Departments of Psychology and Human \& Molecular Genetics, College Behavioral and Emotional Health Institute, Virginia Commonwealth University, Richmond, Virginia. ${ }^{317}$ Broad Institute of MIT and Harvard, Cambridge, USA. ${ }^{318}$ NORMENT, Div. of Mental Health and Addiction, University of Oslo, Oslo, Norway. ${ }^{319}$ Oslo University Hospital, Oslo, Norway. ${ }^{320}$ Department of Psychology, University of Oslo, Norway. ${ }^{321} \mathrm{~K}$. G. Jebsen Centre for Research on
Neuropsychiatric Disorders, University of Bergen, Bergen, Norway. ${ }^{322}$ Department of Biological and Medical Psychology, University of Bergen, Bergen, Norway. ${ }^{323}$ NORMENT, K.G. Jebsen Center for Psychosis Research, Department of Clinical Science, University of Bergen, Norway. ${ }^{324}$ Department of Medical Genetics, Haukeland University Hospital, Bergen, Norway. ${ }^{325}$ Department of Psychiatry, Icahn School of Medicine at Mount Sinai, New York, NY, USA ${ }^{326}$ Seaver Autism Center for Research and Treatment, Icahn School of Medicine at Mount Sinai, New York, NY, USA. ${ }^{327}$ Department of Genetics and Genomic Sciences, and Institute for Genomics and Multiscale Biology, Icahn School of Medicine at Mount Sinai, New York, NY, USA. ${ }^{328}$ The Mindich Child Health \& Development Institute, Icahn School of Medicine at Mount Sinai, New York, NY, USA. ${ }^{329}$ Friedman Brain Institute, Icahn School of Medicine at Mount Sinai, New York, NY, USA. ${ }^{330}$ McLaughlin Centre and Department of Molecular Genetics, University of Toronto, Toronto, Canada. ${ }^{331}$ The Centre for Applied Genomics, Hospital for Sick Children, Toronto, Canada. ${ }^{332}$ Biopsychosocial Corporation, Vienna, Austria. ${ }^{333}$ Department of Psychiatry and Psychotherapy, Medical University of Vienna, Vienna, Austria. ${ }^{334}$ Zentren für Seelische Gesundheit, BBRZ-Med, Vienna, Austria. ${ }^{335}$ Institute of Environmental Medicine, Karolinska Institutet, Stockholm, Sweden. ${ }^{336}$ Rheumatology Unit, Department of Medicine, Karolinska Institutet and Karolinska University Hospital, Solna, Sweden. ${ }^{337}$ Weill Cornell Medical College, New York, New York, USA. ${ }^{338}$ School of Medicine, University of North Dakota, Grand Forks, ND, USA. ${ }^{339}$ Neuropsychiatric Research Institute, Fargo, ND, USA. ${ }^{340}$ Department of Psychiatry \& Biobehavioral Sciences, Semel Institute for Neuroscience \& Human Behavior, David Geffen School of Medicine, University of California at Los Angeles, Los Angeles, CA, USA. ${ }^{341}$ BioRealm, Walnut, California, USA. ${ }^{342}$ Oregon Research Institute, Eugene, OR, USA. ${ }^{343}$ Department of Psychiatry, University of California San Diego, La Jolla, CA, USA. ${ }^{344}$ Centro de Investigación Biomédica en Red de Enfermedades Raras (CIBERER), Instituto de Salud Carlos III, Madrid, Spain. ${ }^{345}$ Department of Psychiatry, Hospital Universitari Vall d'Hebron, Barcelona, Spain. ${ }^{346}$ Psychiatric Genetics Unit, Group of Psychiatry, Mental Health and Addiction, Vall d'Hebron Research Institute (VHIR), Universitat Autònoma de Barcelona, Barcelona, Spain. ${ }^{347}$ Department of Psychiatry and Legal Medicine, Universitat Autònoma de Barcelona, Barcelona, Spain. ${ }^{348}$ Biomedical Network Research Centre on Mental Health (CIBERSAM), Instituto de Salud Carlos III, Madrid, Spain.

${ }^{349}$ Universitat Autònoma de Barcelona, Barcelona, Spain. ${ }^{350}$ Programa Corporatiu "Neurodevelopment Disorders along Life Span", Institut Català de la Salut, Barcelona, Spain. ${ }^{351}$ Clinica Galatea y PAIMM, Mental Health Program for Impaired Physicians, Barcelona, Spain. ${ }^{352} \mathrm{Child}$ and Adolescent Mental Health Unit, Hospital Universitario Mútua de Terrassa, Barcelona, Spain. ${ }^{353}$ Fundació Docència i Recerca Mútua Terrassa. ${ }^{354}$ K.G. Jebsen Centre for Neuropsychiatric Disorders, Department of Biomedicine, University of Bergen, Norway. ${ }^{355}$ Division of Psychiatry, Haukeland University Hospital, Bergen, Norway. ${ }^{356}$ K.G. Jebsen Centre for Neuropsychiatric Disorders, Department of Clinical Science, University of Bergen, Norway. ${ }^{35}$ Institute of Medical Informatics and Statistics, Kiel University, Kiel, Germany. ${ }^{358}$ Child and Adolescent Psychiatry/Psychotherapy, University Medical Center, Goettingen, Germany. ${ }^{359}$ Institute of Psychiatry, Psychology and Neuroscience, King's College London, London, UK. ${ }^{360}$ Clinic for Child and Adolescent Psychiatry and Psychotherapy, University of Duisburg-Essen, Essen, Germany. ${ }^{361}$ Child and Adolescent Psychiatry and Psychotherapy, Central Institute of Mental Health, Medical Faculty Mannheim, University of Heidelberg, Mannheim, Germany. ${ }^{362}$ Department of Human Genetics, Radboud University Medical Center, Nijmegen, The Netherlands. ${ }^{363}$ Department of Psychiatry, Radboud University Medical Center, Nijmegen, The Netherlands. ${ }^{364}$ Donders Institute for Brain, Cognition and Behaviour, Radboud University, Nijmegen, The Netherlands. ${ }^{365}$ Department of Cognitive Neuroscience, Donders Institute for Brain, Cognition and Behavior, Radboud University Medical Centre, Nijmegen, The Netherlands. ${ }^{366}$ Karakter Child and Adolescent Psychiatry University Center, Nijmegen, The Netherlands. ${ }^{367}$ Department of Psychiatry \& Human Genetics, Donders Institute for Brain, Cognition and Behaviour, Radboud University Medical Center, Nijmegen, The Netherlands. ${ }^{368}$ Department of Psychiatry,

Psychosomatic Medicine and Psychotherapy, University Hospital Frankfurt, Frankfurt am Main, Germany. ${ }^{369}$ Laboratory of Psychiatric Neurobiology, Institute of Molecular Medicine, I.M. Sechenov First Moscow State Medical University, Moscow, Russia. ${ }^{370}$ Department of Translational Psychiatry, School for Mental Health and Neuroscience (MHeNS), Maastricht University, Maastricht, The Netherlands.

${ }^{371}$ Division of Molecular Psychiatry, Center of Mental Health, University of Wuerzburg, Wuerzburg, Germany. ${ }^{372}$ Department of Child and Adolescent Psychiatry, Psychosomatics and Psychotherapy, University Hospital Frankfurt, Goethe University, Frankfurt am Main, Germany.

${ }^{373}$ Center of Mental Health, Department of Child and Adolescent 
Psychiatry, Psychosomatics and Psychotherapy, University Hospital of Wuerzburg, Wuerzburg, Germany. ${ }^{374}$ School of Psychology, Cardiff University, UK. ${ }^{375}$ Central Institute of Mental Health, Department of Genetic Epidemiology in Psychiatry, Medical Faculty Mannheim, University of Heidelberg, Mannheim, Germany. ${ }^{376}$ National Centre for Register-based Research, Aarhus University, Aarhus, Denmark. ${ }^{377}$ Hospital of Telemark, Kragerø, Norway. ${ }^{378}$ Department of Biomedicine and Human Genetics, Aarhus University, Aarhus, Denmark. ${ }^{379}$ Center for Integrative Sequencing (iSEQ), Aarhus University, Aarhus, Denmark. ${ }^{380}$ Aarhus Genome Center, Aarhus, Denmark. ${ }^{381}$ Department of Psychology, Emory University, Atlanta, GA, USA. ${ }^{382}$ Department of Medical Informatics and Clinical Epidemiology, Oregon Health \& Science University, Portland, OR, USA. ${ }^{383}$ Department of Psychological and Brain Sciences, University of lowa, lowa City, IA, USA. ${ }^{384}$ Departamento de Genética, Universidade Federal do Rio Grande do Sul, Porto Alegre, Brazil. ${ }^{385}$ ADHD Outpatient Clinic, Hospital de Clínicas de Porto Alegre, Porto Alegre, Brazil. ${ }^{386}$ Neurosciences and Mental Health Program, Research Institute, Hospital for Sick Children, Toronto, Canada. ${ }^{387}$ University of Toronto, Toronto, Canada. ${ }^{388}$ Hospital for Sick Children, Toronto, Canada. ${ }^{389}$ Department of Psychiatry, University of California, Los Angeles, Los Angeles, CA, USA. ${ }^{390}$ Semel Institute for Neuroscience \& Human Behavior, David Geffen School of Medicine, University of California at Los Angeles, Los Angeles, CA, USA. ${ }^{391}$ ADHD Outpatient Clinic, Hospital de Clínicas de Porto Alegre, Porto Alegre, Brazil. ${ }^{392}$ Department of Psychiatry, Faculdade de Medicina, Universidade Federal do Rio Grande do Sul, Porto Alegre, Brazil. ${ }^{393}$ Department of Psychiatry, University of California, San Francisco, San Francisco, CA USA. ${ }^{394}$ Institute for Human Genetics, University of California, San Francisco, San Francisco, CA, USA. ${ }^{395}$ Weill Institute for Neurosciences, University of California, San Francisco, San Francisco, CA, USA. ${ }^{396}$ Department of Pharmacy and Biotechnology, University of Bologna, Bologna, Italy. ${ }^{397}$ Department of Psychiatry, University of British Columbia, Vancouver, Canada. ${ }^{398}$ Institute of Mental Health, University of British Columbia, Vancouver, Canada. ${ }^{399}$ Stella Maris Clinical Research Institute for Child and Adolescent Neuropsychiatry, Pisa, Italy. ${ }^{400}$ Sorbonne Université, INSERM, CNRS, Neuroscience Paris Seine, Institut de Biologie Paris Seine, Paris, France. ${ }^{401}$ NIHR Biomedical Research Centre in Mental Health Maudsley Hospital, London, UK. ${ }^{402}$ Department of Human Genetics, David Geffen School of Medicine, University of California at Los Angeles, Los Angeles, CA, USA. ${ }^{403}$ LifeOmic, Indianapolis, IN, USA. ${ }^{404}$ Department of Psychiatry and Behavioral Sciences, Duke University, Durham, NC, USA. ${ }^{405}$ University Clinic of Pediatrics, Faculty of Medicine, University of Coimbra, Coimbra, Portugal. ${ }^{406}$ Child Developmental Center, Hospital Pediátrico, Centro Hospitalar e Universitário de Coimbra, Coimbra, Portugal. ${ }^{407}$ Mindich Child Health and Development Institute, Icahn School of Medicine at Mount Sinai, New York, NY, USA. ${ }^{408}$ Dept of Clinical Genetics, Our Lady's Children's Hospital, Crumlin, Dublin, Ireland. ${ }^{409}$ School of Medicine and Medical Science, University College Dublin, Dublin, Ireland. ${ }^{410}$ Division of Molecular Genome Analysis and Division of Cancer Genome Research, German Cancer Research Center (DKFZ), Heidelberg, Germany. ${ }^{411}$ Inserm U955, Psychiatrie Translationnelle, Créteil, France. ${ }^{412}$ Faculté de Médecine, Université Paris Est, Créteil, France. ${ }^{413}$ Fondation FondaMental, Créteil, France. ${ }^{414}$ Children's Hospital Los Angeles, Los Angeles, CA, USA. ${ }^{415}$ Yale Center for Genome Analysis, Yale University, New Haven, CT, USA. ${ }^{416}$ Department of Genetics, Yale University School of Medicine, New Haven, CT, USA. ${ }^{417}$ Division of Child and Adolescent Psychiatry, Department of Psychiatry and Human Behavior, Brown University, Providence, RI, USA. ${ }^{418}$ Institute of Neuroscience, Newcastle University, Newcastle, UK. ${ }^{419}$ Newcastle Upon Tyne Hospitals NHS Foundation Trust, Newcastle, UK. ${ }^{420}$ Northumberland, Tyne \& Wear NHS Foundation Trust, Northumberland, UK. ${ }^{421}$ Genomics Medicine Ireland, Dublin, Ireland. ${ }^{422}$ Department of Medical Epidemiology and Biostatistics, Karolinska Institutet, Stockholm, Sweden. ${ }^{423}$ Department of Psychiatry, Hospital for Sick Children and University of Toronto, Toronto, Canada. ${ }^{424}$ Program in Genetics and Genome Biology, Hospital for Sick Children, Toronto, Canada. ${ }^{425}$ Department of Psychiatry, Carver College of Medicine, University of lowa, lowa City, IA, USA. ${ }^{426}$ Division of Medical Genetics, Department of Medicine, University of Washington, Seattle, WA, USA. ${ }^{427}$ Department of Psychiatry, University of Illinois at Chicago, Chicago, IL, USA. ${ }^{428}$ Tufts University School of Medicine, Portland, ME, USA. ${ }^{429}$ Center for Psychiatric Research, Maine Medical Center Research Institute, Portland, ME, USA. ${ }^{430}$ Department of Psychiatry, Tufts University School of Medicine, Boston, MA, USA. ${ }^{431}$ Child and Adolescent Psychiatry Department, Robert Debre Hospital, APHP, Paris, France. ${ }^{432}$ Human Genetics and Cognitive Functions, Institut Pasteur, Paris, France. ${ }^{433}$ Centre d'Etudes et de Recherches en Psychopathologie et Psychologie de la Santé (CERPPS), Université Toulouse Jean Jaurès, Toulouse, France. ${ }^{434}$ CERESA, Toulouse, France. ${ }^{435}$ Institut Universitaire de France, Paris, France. ${ }^{436}$ Academic Centre on Rare
Diseases University College Dublin (ACoRD/UCD), Dublin, Ireland ${ }^{437}$ McKusick-Nathans Institute of Genetic Medicine, Johns Hopkins University School of Medicine, Baltimore, MD, USA. ${ }^{438}$ Institute of Psychiatric Phenomics and Genomics (IPPG), University Hospital, LMU Munich, Munich, Germany. ${ }^{439}$ Department of Psychiatry and Psychotherapy, University Medical Center Göttingen, Göttingen, Germany. ${ }^{440}$ Department of Psychiatry and Behavioral Sciences, Johns Hopkins University, Baltimore, MD, USA. ${ }^{441}$ Human Genetics Branch, National Institute of Mental Health, National Institutes of Health, US Department of Health and Human Services, Bethesda, MD, USA. ${ }^{442}$ Molecular and Behavioral Neuroscience Institute, University of Michigan, Ann Arbor, MI, USA. ${ }^{443}$ Department of Psychiatry, University of Michigan, Ann Arbor, MI, USA. ${ }^{444}$ SRH University Heidelberg. Academy for Psychotherapy, Heidelberg, Germany. ${ }^{445}$ Division of Neuroscience, School of Medicine, University of Dundee, Dundee, UK. ${ }^{446}$ Advanced Interventions Service, NHS Tayside, Dundee, UK ${ }^{447}$ NORMENT, K.G. Jebsen Centre for Psychosis Research, Institute of Clinical Medicine, University of Oslo, Oslo, Norway. ${ }^{448}$ Division of Mental Health and Addiction, Oslo University Hospital, Oslo, Norway. ${ }^{449}$ Cognitive Genetics and Cognitive Therapy Group, Neuroimaging, Cognition and Genomics (NICOG) Centre, School of Psychology and Discipline of Biochemistry, National University of Ireland Galway, Galway, Ireland. ${ }^{450}$ Division of Psychiatry, University of Edinburgh. Edinburgh, UK. ${ }^{451}$ Department of Molecular Medicine and Surgery, Karolinska Institutet, Stockholm, Sweden. ${ }^{452}$ Center for Molecular Medicine, Karolinska University Hospital, Stockholm, Sweden. ${ }^{453}$ Université Paris Est, Faculté de Médecine, Créteil, France. ${ }^{454}$ Neuroscience Research Australia, Sydney, Australia. ${ }^{455}$ School of Medical Sciences, University of New South Wales, Sydney, Australia. ${ }^{456}$ Unidad de Salud Mental, Hospital Regional Universitario de Malag Malaga, Spain. ${ }^{457}$ Instituto de Investigación Biomédica de Málaga (IBIMA), Malaga, Spain. ${ }^{458}$ Department of Biomedicine, University of Basel, Basel, Switzerland. ${ }^{459}$ Institute of Medical Genetics and Pathology, University Hospital Basel, Basel, Switzerland. ${ }^{460}$ Institute of Neuroscience and Medicine (INM-1), Research Centre Jülich, Jülich, Germany. ${ }^{461}$ Institute of Human Genetics, University of Bonn, Bonn, Germany. ${ }^{462}$ Department of Psychiatry and Psychotherapy, University Hospital Carl Gustav Carus, Technische Universität Dresden, Dresden, Germany. ${ }^{463}$ School of Psychiatry, University of New South Wales, Sydney, Australia. ${ }^{464}$ Black Dog Institute, Sydney, Australia. ${ }^{465}$ University of Chicago, Chicago, IL, USA. ${ }^{466}$ Washington University, St. Louis, MO, USA. ${ }^{467}$ Department of Mental Health, Johns Hopkins Bloomberg School of Public Health, Baltimore, MD, USA. ${ }^{468}$ Department of Psychiatry, Dalhousie University, Halifax, Canada. ${ }^{469}$ National Institute of Mental Health, Klecany, Czech Republic. ${ }^{470}$ Montreal Neurological Institute, McGill University, Montréal, Canada. ${ }^{471}$ Department of Neurology and Neurosurgery, McGill University, Montréal, Canada. ${ }^{472}$ Department of Psychiatry, McGill University, Montréal, Canada. ${ }^{473}$ University College London, London, UK. ${ }^{474}$ Center for Neurobehavioral Genetics, Semel Institute for Neuroscience \& Human Behavior, University of California at Los Angeles, Los Angeles, CA, USA. ${ }^{475}$ UMC Utrecht, Utrecht, The Netherlands. ${ }^{476}$ SUNY Downstate Medical Center, Brooklyn, NY, USA ${ }^{477}$ Hospital for Psychiatry and Psychotherapy, Cologne, Germany. ${ }^{478}$ Laboratory of Psychiatric Genetics, Department of Psychiatry, Poznan University of Medical Sciences, Poznan, Poland. ${ }^{479}$ Douglas Mental Health University Institute, McGill University, Montreal, Canada. ${ }^{480}$ Department of Translational Research in Psychiatry, Max-Planck Institute of Psychiatry, Munich, Germany. ${ }^{481}$ National Centre for Mental Health, MRC Centre for Neuropsychiatric Genetics and Genomics, Cardiff University, Cardiff, UK. ${ }^{482}$ Department Complex Trait Genetics, Center for Neurogenomics and Cognitive Research, Vu University, Amsterdam, The Netherlands. ${ }^{483}$ Department Clinical Genetics, VU University Medical Center, Amsterdam Neuroscience, Amsterdam, The Netherlands. ${ }^{484}$ Department of Neurology, Klinikum rechts der Isar, Technical University of Munich, Munich, Germany. ${ }^{485}$ Institute of Human Genetics, University of Bonn, Bonn, Germany. ${ }^{486}$ Department of Psychiatry (UPK), University of Basel, Basel, Switzerland. ${ }^{487}$ Discipline of Psychiatry, University of Adelaide, Adelaide, Australia. ${ }^{488}$ Queensland Brain Institute, University of Queensland, Brisbane, Australia. ${ }^{489}$ Bela Menso Brain and Behaviou Centre, James Cook University, Varsity Lakes, Australia. ${ }^{490}$ Bond University, Faculty of Society and Design, Robina, Australia. ${ }^{491}$ Division of Psychiatry, Centre for Clinical Brain Sciences, University of Edinburgh, Edinburgh, UK. ${ }^{492}$ Centre for Genomic and Experimental Medicine, University of Edinburgh, Edinburgh, UK. ${ }^{493}$ Interfaculty Institute for Genetics and Functional Genomics, University Medicine Greifswald, Greifswald, Germany. ${ }^{494}$ Department of Biochemistry and Molecular Biology II, Institute of Neurosciences, Center for Biomedical Research, University of Granada, Granada, Spain. ${ }^{495}$ Bioinformatics Research Centre, Aarhus University, Aarhus, Denmark. ${ }^{496}$ Child Health Research Centre, University of Queensland, Brisbane, Australia. ${ }^{497}$ Child and Youth Mental Health Service, Children's Health
Queensland Health and Hospital Service, Brisbane, Australia. ${ }^{498}$ Brain and Mind Centre, University of Sydney, Sydney, Australia. ${ }^{499}$ School of Psychology and Counselling, Faculty of Health, Institute of Health and Biomedical Innovation, Queensland University of Technology, Queensland, Australia. ${ }^{500}$ University of Queensland, Brisbane, Australia. ${ }^{501}$ Department of Psychiatry, Harvard Medical School, Boston, MA, USA. ${ }^{502}$ Amsterdam Public Health Research Institute, VU Medical Center, Amsterdam, the Netherlands. ${ }^{503}$ Department of Research and Innovation, GGZ Ingeest, Specialized Mental Health Care, Amsterdam, the Netherlands. ${ }^{504}$ Janssen Research \& Development LLC, Titusville, NJ, USA. ${ }^{505}$ Institute of Clinical Chemistry and Laboratory Medicine, University Medicine Greifswald, Greifswald, Germany. ${ }^{506}$ German Centre for Cardiovascular Research (DZHK e.V.), Partner Site Greifswald, Greifswald, Germany. ${ }^{507}$ Research School of Behavioural and Cognitive Neurosciences, Department of Psychiatry, University Medical Center Groningen, University of Groningen, Groningen, the Netherlands. ${ }^{508}$ Department of Psychiatry GGZ INGEEST, Amsterdam, the Netherlands.

${ }^{509}$ Department of Cell Biology, SUNY Downstate Medical Center, Brooklyn, NY, USA. ${ }^{510}$ Mathison Centre for Mental Health Research \& Education, Hotchkiss Brain Institute, Cumming School of Medicine, University of Calgary, Calgary, Canada. ${ }^{511}$ Departments of Psychiatry and Medical Genetics, Cumming School of Medicine, University of Calgary, Calgary, Canada. ${ }^{512}$ Krembil Research Institute, University Health Network, Toronto, Canada. ${ }^{513}$ Grupo de Genética Molecular. Instituto de Biología, Facultad de Ciencias Exactas y Naturales, Universidad de Antioquia, Medellín, Colombia. ${ }^{514}$ Johns Hopkins University School of Medicine, Baltimore, MD, USA. ${ }^{515}$ Department of Psychiatry, Sao Paulo Medical School, University of Sao Paulo, Sao Paulo, Brazil. ${ }^{516}$ Depto. Farmacogenética, Instituto Nacional de Psiquiatria Ramon de la Fuente Muñiz, Mexico City, Mexico.

${ }^{517}$ University of Groningen, Groningen, the Netherlands. ${ }^{518}$ Department of Psychiatry, University of Groningen and University Medical Center, Groningen, the Netherlands. ${ }^{519}$ Department of Specialized Trainings, GGZ Drenthe Mental Health Care Services, Assen, the Netherlands. ${ }^{520}$ Ospedale San Raffaele, Milano, Italy. ${ }^{521}$ Bio4Dreams Srl, Milan, Italy. ${ }^{522}$ University of California, San Francisco, CA, USA. ${ }^{523}$ Yale University School of Medicine, New Haven, CT, USA. ${ }^{524}$ Department of Psychiatry, Academic Medical Center, University of Amsterdam, Amsterdam, the Netherlands. ${ }^{525}$ Netherlands Institute for Neuroscience, Royal Netherlands Academy of Arts and Sciences, Amsterdam, the Netherlands. ${ }^{526}$ Department of Child and Adolescent Psychiatry, University Medical Center Groningen, University of Groningen, Groningen, The Netherlands. ${ }^{527}$ Centre National Maladie 'Syndrome Rare Gilles de la Tourette', Groupe Hospitalier Pitié-Salpêtrière, Paris, France. ${ }^{528}$ Assistance Publique-Hôpitaux de Paris, Départment de Neurologie, Groupe Hospitalier Pitié-Salpêtrière, Paris, France. ${ }^{529}$ Sorbonne Universités, UPMC Université Paris 06, UMR S 1127, CNRS UMR 7225, ICM, Paris, France. ${ }^{530}$ Bioinformatics Interdepartmental Program, University of California, Los Angeles, LoS Angeles, CA, USA. ${ }^{531}$ De Bascule, Amsterdam, The Netherlands.

${ }^{532}$ Department of Child and Adolescent Psychiatry, Academic Medical Center, University of Amsterdam, Amsterdam, the Netherlands.

${ }^{533}$ Carver College of Medicine, University of lowa, lowa City, IA, USA

${ }^{534}$ MRC Unit on Risk \& Resilience in Mental Disorders, Department of Psychiatry, University of Cape Town, Cape Town, South Africa.

${ }^{535}$ Cold Spring Harbor Laboratory, Cold Spring Harbor, NY, USA

${ }^{536}$ Department of Psychiatry and Human Behavior, University of California, Irvine, Irvine, CA, USA. ${ }^{537}$ Department of Neurology, University of Florida, Gainesville, FL, USA. ${ }^{538}$ Sección de Neuropediatría, Instituto de Biomedicina de Sevilla, Hospital Universitario Virgen del Rocío/CSIC/Universidad de Sevilla, Seville, Spain. ${ }^{539}$ Yulius Academy, Yulius Mental Health Organization, Barendrecht, The Netherlands. ${ }^{540}$ Department of Psychology, University of Denver, Denver, CO, USA. ${ }^{541}$ Faculdade de Medicina FMUSP, Universidade de São Paulo, São Paulo, Brazil. ${ }^{542}$ Unidad de Trastornos del Movimiento, Servicio de Neurología y Neurofisiología Clínica, Instituto de Biomedicina de Sevilla, Hospital Universitario Virgen del Rocío/CSIC/Universidad de Sevilla, Seville, Spain. ${ }^{543}$ Centro de Investigación Biomédica en Red sobre Enfermedades Neurodegenerativas (CIBERNED), Madrid, Spain. ${ }^{544}$ National Institute of Genomic Medicine (INMEGEN), Ciudad de México, Mexico.

${ }^{545}$ Clinical Research, Grupo Médico Carracci, Mexico City, Mexico. ${ }^{546}$ Departments of Neurology and Neurosurgery, University of Florida, Gainesville, FL, USA. ${ }^{547}$ Fixel Center for Neurological Diseases, University of Florida, Gainesville, FL, USA. ${ }^{548}$ McKnight Brain Institute, University of Florida, Gainesville, FL, USA. ${ }^{549}$ Department of Psychiatry, Yale School of Medicine, New Haven, CT, USA. ${ }^{550}$ Department of Biological Sciences, Purdue University, West Lafayette, Indiana, USA. ${ }^{551}$ Division of Adolescent and Child Psychiatry, Department of Psychiatry, Lausanne University Hospital, Lausanne, Switzerland. ${ }^{552} \mathrm{Child}$ and Adolescent Mental Health Centre, Mental Health Services Capital Region Copenhagen, University of 
Copenhagen, Copenhagen, Denmark. ${ }^{553}$ Moscow Institute of Physics and Technology, Dolgoprudny, Institusky 9, Moscow, Russia. ${ }^{554}$ Department of Psychiatry. David Geffen School of Medicine, University of California at Los Angeles, Los Angeles, CA, USA. ${ }^{555}$ Frederick W. Thompson Anxiety Disorders Centre, Sunnybrook Health Sciences Centre, Toronto, Canada. ${ }^{556}$ Department of Psychiatry, University of Toronto, Toronto, Canada. ${ }^{557}$ Division of Neuropsychiatry, University College London, London, UK. ${ }^{558}$ Department of Child and Adolescent Psychiatry, Faculty of Medicine, Technischen Universität Dresden, Dresden, Germany. ${ }^{559} \mathrm{Child}$ and Adolescent Psychiatry Unit (UPIA), Department of Psychiatry, Federal University of São Paulo, Brazil. ${ }^{560}$ Yale Child Study Center, Yale University School of Medicine, New Haven, CT, USA. ${ }^{561}$ University Health Network, University of Toronto, Toronto, Canada. ${ }^{562}$ Youthdale Treatment Centers, Toronto, Canada. ${ }^{563}$ Groote Schuur Hospital, Cape Town, South Africa. ${ }^{564}$ Department of Molecular Biology and Genetics, Democritus University of Thrace, Alexandroupolis, Greece. ${ }^{565}$ Laboratory of Pharmaceutical Biotechnology, Ghent University, Ghent, Belgium. ${ }^{566}$ Pfizer, Inc., New York, NY, USA. ${ }^{567}$ Department of Child Psychiatry, Medical University of Warsaw, Warsaw, Poland. ${ }^{568}$ Sorbonne Université, Faculty of Médecine, Paris, France. ${ }^{569}$ Reference center for Gilles de la Tourette syndrome, Pitie-Salpetriere Hospital, Paris, France. ${ }^{570}$ Department of Physiology, Saint Antoine Hospital, Paris, France. ${ }^{571}$ Butler Hospital, Providence, RI, USA. ${ }^{572}$ Alpert Medical School of Brown University, Providence, RI, USA. ${ }^{573}$ Department of Psychiatry and Psychotherapy, University Medicine Greifswald, Greifswald, Germany. ${ }^{574}$ Institute of Human Genetics, University Hospital Essen, University Duisburg Essen, Essen, Germany. ${ }^{575}$ INSERM, U 1127, CNRS UMR 7225, Sorbonne Universités, UPMC Univ Paris 06 UMR S 1127, Paris, France. ${ }^{576}$ IGBMC, CNRS UMR 7104/INSERM U964/Université de Strasbourg, IIIkirch, France. ${ }^{577}$ Vanderbilt University Medical Center, Nashville, TN, USA. ${ }^{578}$ Escuela de Ciencias de la Salud, Universidad Pontificia Bolivariana, Medellín, Colombia. ${ }^{579}$ Laboratorio de Genética Molecular. SIU, Universidad de Antioquia, Medellín, Colombia. ${ }^{580}$ School of Nursing, Louisiana State University Health Sciences Center, New Orleans, LA, USA. ${ }^{581}$ Brain Center Rudolf Magnus, University Medical Center Utrecht, Utrecht, The Netherlands. ${ }^{582} \mathrm{School}$ of Biomedical Sciences and Pharmacy, The University of Newcastle, Callaghan, Australia. ${ }^{583}$ Priority Research Centre for Brain and Mental Health Research, Hunter Medical Research Institute, Newcastle, Australia. ${ }^{584}$ Schizophrenia Research Institute, Sydney, Australia. ${ }^{585}$ Institute of Mental Health, Singapore, Singapore. ${ }^{586}$ Assistance Publique Hopitaux de Paris, GH Pitié-Salpêtrière, Paris, France. ${ }^{587}$ Sorbonne Université, CNRS UMR 7222 Institut des Systèmes Intelligents et Robotiques, Paris, France. ${ }^{588}$ Departments of Medicine and Psychiatry, School of Medicine University of Cantabria-IDIVAL, University Hospital Marqués de Valdecilla, Santander, Spain. ${ }^{589}$ Minerva Neurosciences Inc., Waltham, MA, USA. ${ }^{590}$ Sackler School of Medicine, Tel Aviv University, Tel Aviv, Israel. ${ }^{591}$ VA Boston Healthcare System, Boston, MA, USA. ${ }^{592}$ APC Microbiome Ireland,
University College Cork, Cork, Ireland. ${ }^{593}$ Department of Psychiatry, University College Cork, Cork, Ireland. ${ }^{594}$ Neuroimaging, Cognition and Genomics (NICOG) Centre, School of Psychology, National University of Ireland Galway, Galway, Ireland. ${ }^{595}$ Center for Psychiatric Genetics, NorthShore University HealthSystem Research Institute, Evanston, IL, USA. ${ }^{596}$ Department of Psychiatry and Behavioral Neuroscience, University of Chicago, Chicago, IL, USA. ${ }^{597}$ Arkin, Amsterdam, the Netherlands. ${ }^{598}$ Department for Congenital Disorders, Statens Serum Institut, Copenhagen, Denmark. ${ }^{599}$ Department of Medical Genetics, Medical University, Sofia, Bulgaria. ${ }^{600}$ Department of Molecular Bases of Human Genetics, Institute of Molecular Genetics, Russian Academy of Sciences, Moscow, Russia. ${ }^{601}$ Latvian Biomedical Research and Study Centre, Riga, Latvia. ${ }^{602}$ Vilnius University, Vilnius, Lithuania. ${ }^{603}$ Institute of Mental Health, Lee Kong Chian School of Medicine, Nanyang Technological University, Singapore, Singapore. ${ }^{604}$ Department of Human Genetics, Institute of Molecular Genetics, Russian Academy of Sciences, Moscow, Russia. ${ }^{605}$ Hunter New England Local Health District, Newcastle, Australia. ${ }^{606}$ Department of Psychiatry, University of Helsinki, Helsinki, Finland. ${ }^{607}$ Department of Psychiatry, Psychosomatics and Psychotherapy, Center of Mental Health, University Hospital Wuerzburg, Wuerzburg, Germany. ${ }^{608}$ Department of Biomedicine, Aarhus University, Aarhus, Denmark. ${ }^{609}$ Department of Clinical Neuroscience, Centre for Psychiatry Research, Karolinska Institutet, Stockholm, Sweden. ${ }^{610}$ Centre for Neuroimaging and Cognitive Genomics (NICOG), National University of Ireland, Galway, Galway, Ireland. ${ }^{611}$ NCBES Galway Neuroscience Centre, National University of Ireland, Galway, Galway, Ireland. ${ }^{612}$ Department of Psychiatry, Royal College of Surgeons in Ireland, Dublin, Ireland. ${ }^{613}$ Philipps-Universität Marburg and Marburg University Hospital UKGM, Marburg, Germany. ${ }^{614}$ Department of Psychiatry and Psychotherapy, Jena University Hospital, Jena, Germany. ${ }^{615}$ Maastricht University Medical Centre, Maastricht, the Netherlands. ${ }^{616}$ Department of Psychosis Studies, Institute of Psychiatry, King's College London, London, UK. ${ }^{617}$ Melbourne Neuropsychiatry Centre, Department of Psychiatry, University of Melbourne \& Melbourne Health, Victoria, Australia. ${ }^{618}$ Centre for Neural Engineering, Department of Electrical and Electronic Engineering, University of Melbourne, Victoria, Australia. ${ }^{619}$ Oxford Health NHS Foundation Trust, Oxford, UK. ${ }^{620}$ Department of Psychiatry, University of Oxford, Oxford, UK. ${ }^{621}$ Department of Psychiatry and Behavioral Sciences, NorthShore University HealthSystem Research Institute, Evanston, IL, USA. ${ }^{622}$ Faculty of Science, Medicine and Health, University of Wollongong, Wollongong, Australia. ${ }^{623}$ Yong Loo Lin School of Medicine, National University of Singapore, Singapore. ${ }^{624}$ Lee Kong Chian School of Medicine, Nanyang Technological University, Singapore, Singapore. ${ }^{625}$ School of Biomedical Sciences, Chinese University of Hong Kong, Shatin, Hong Kong. ${ }^{626} \mathrm{KIZ}$-CUHK Joint Laboratory of Bioresources and Molecular Research of Common Diseases, Kunming Institute of Zoology, Chinese Academy of Sciences, Kunming, China. ${ }^{627}$ Chinese University of Hong Kong, Hong Kong. ${ }^{628}$ Sheba Medical Center,
Ramat Gan, Israel. ${ }^{629}$ Departments of Psychiatry and Genetics, Washington University School of Medicine, St. Louis, MO, USA ${ }^{630}$ UCL Genetics Institute, University College London, London, UK. ${ }^{631}$ Centre for Psychiatry, Barts and the London School of Medicine and Dentistry, London, UK. ${ }^{632}$ School of Medicine \& Public Health, University of Newcastle, Callaghan, Australia. ${ }^{633}$ Priority Research Centre for Health Behaviour, University of Newcastle, Callaghan, Australia. ${ }^{634}$ Research Unit, Sørlandet Hospital, Kristiansand, Norway. ${ }^{635}$ Department of Statistics and Applied Probability, University of California, Santa Barbara, CA, USA. ${ }^{636}$ Computational Research Division, Lawrence Berkeley National Laboratory, University of California at Berkeley, Berkeley, CA, USA. ${ }^{637} \mathrm{NSW}$ Health Pathology, Newcastle, Australia. ${ }^{638}$ Virginia Institute for Psychiatric and Behavioral Genetics, Department of Psychiatry, Virginia Commonwealth University, Richmond, VA, USA. ${ }^{639}$ Institute of Psychiatry, Psychology \& Neuroscience, Social Genetics \& Developmental Psychiatry Center, MRC, Kings College London, London, UK. ${ }^{640}$ NIHR Maudsley Biomedical Research Centre, South London \& Maudsley NHS Trust \& King's College London, London, UK. ${ }^{641}$ Departments of Psychiatry and Nutrition, University of North Carolina at Chapel Hill, Chapel Hill, NC, USA. ${ }^{642}$ Departments of Psychiatry and of Neuroscience and Physiology, SUNY Upstate Medical University, Syracuse, NY, USA. ${ }^{643}$ Department of Psychiatry, Virginia Commonwealth University, Richmond, VA, USA.

${ }^{644}$ Division Biomedical Genetics, University Medical Center Utrecht, Utrecht, the Netherlands. ${ }^{645}$ Department of Psychiatry and UF Genetics Institute, University of Florida, Gainesville, FL, USA. ${ }^{646}$ Division of Cognitive and Behavioral Neurology, Brigham and Women's Hospital, Boston, MA, USA. ${ }^{647}$ Department of Neurology, Yale School of Medicine, New Haven, CT, USA. ${ }^{648}$ Department of Genetics and Psychiatry, University of North Carolina School of Medicine, Chapel Hill, North Carolina, USA. ${ }^{649}$ Neuropsychiatric Genetics Research Group, Department of Psychiatry, Trinity College Dublin, Dublin, Ireland.

*Corresponding author. Email: verneri.anttila@gmail.com (V.A.) acorvin@tcd.ie (A.C.); bneale@broadinstitute.org (B.M.N.) †These authors contributed equally to this work.

\section{SUPPLEMENTARY MATERIALS}

www.sciencemag.org/content/360/6395/eaap8757/suppl/DC1 Materials and Methods

Supplementary Text

Consortium Memberships

Figs. S1 to S10

Tables S1 to 13

References (68-108)

15 April 2016; resubmitted 7 February 2017

Accepted 24 April 2018

10.1126/science.aap8757 
Science (print ISSN 0036-8075; online ISSN 1095-9203) is published by the American Association for the Advancement of Science, 1200 New York Avenue NW, Washington, DC 20005. 2017 () The Authors, some rights reserved; exclusive licensee American Association for the Advancement of Science. No claim to original U.S. Government Works. The title Science is a registered trademark of AAAS. 
Science (print ISSN 0036-8075; online ISSN 1095-9203) is published by the American Association for the Advancement of Science, 1200 New York Avenue NW, Washington, DC 20005. 2017 () The Authors, some rights reserved; exclusive licensee American Association for the Advancement of Science. No claim to original U.S. Government Works. The title Science is a registered trademark of AAAS. 